Wright State University

CORE Scholar

$9-1-2008$

\title{
Variation of the Martian Ionospheric Electron Density from Mars Express Radar Soundings
}

D. D. Morgan

D. A. Gurnett

D. L. Kirchner

Jane L. Fox

Wright State University - Main Campus, jane.fox@wright.edu

E. Nielsen

See next page for additional authors

Follow this and additional works at: https://corescholar.libraries.wright.edu/physics

Part of the Astrophysics and Astronomy Commons, and the Physics Commons

\section{Repository Citation}

Morgan, D. D., Gurnett, D. A., Kirchner, D. L., Fox, J. L., Nielsen, E., \& Plaut, J. J. (2008). Variation of the Martian Ionospheric Electron Density from Mars Express Radar Soundings. Journal of Geophysical Research-Space Physics, 113, A09303.

https://corescholar.libraries.wright.edu/physics/27

This Article is brought to you for free and open access by the Physics at CORE Scholar. It has been accepted for inclusion in Physics Faculty Publications by an authorized administrator of CORE Scholar. For more information, please contact library-corescholar@wright.edu. 


\section{Authors}

D. D. Morgan, D. A. Gurnett, D. L. Kirchner, Jane L. Fox, E. Nielsen, and J. J. Plaut

This article is available at CORE Scholar: https://corescholar.libraries.wright.edu/physics/27 


\title{
Variation of the Martian ionospheric electron density from Mars Express radar soundings
}

\author{
D. D. Morgan, ${ }^{1}$ D. A. Gurnett, ${ }^{1}$ D. L. Kirchner, ${ }^{1}$ J. L. Fox, ${ }^{2}$ E. Nielsen, ${ }^{3}$ and J. J. Plaut ${ }^{4}$ \\ Received 16 April 2008; revised 16 April 2008; accepted 23 May 2008; published 5 September 2008.
}

[1] The Mars Advanced Radar for Subsurface and Ionospheric Sounding aboard Mars Express has been in operation for over 2 years. Between 14 August 2005 and 31 July 2007, we obtain 34,492 ionospheric traces, of which 14,060 yield electron density profiles and 12,291 yield acceptable fits to the Chapman ionospheric model. These results are used to study the Martian ionosphere under changing conditions: the presence or absence of solar energetic particles, solar EUV flux, season, solar zenith angle, and latitude. The 2-year average subsolar maximum electron density $n_{0}$ is $1.62 \times 10^{5} \mathrm{~cm}^{-3}$, the average subsolar electron density altitude $h_{0}$ is $128.2 \mathrm{~km}$, and the average neutral scale height $H$ is $12.9 \mathrm{~km}$. Solar energetic particle events are associated with a $6 \%$ increase in $n_{0}$, a $3 \mathrm{~km}$ decrease in $h_{0}$, and a $0-7 \mathrm{~km}$ decrease in $H$. The value of $n_{0}$ varies smoothly between $1.4 \times 10^{5}$ and $1.8 \times$ $10^{5} \mathrm{~cm}^{-3}$, yielding $d \ln n_{0} / d \ln \mathrm{F} 10.7=0.30 \pm 0.4 ; h_{0}$ varies between 115 and $135 \mathrm{~km}$, while $H$ remains relatively constant with EUV flux and season, in contrast with previous work. The value of $h_{0}$ decreases toward the terminator at low latitude but increases poleward during summer; $H$ varies from $11 \mathrm{~km}$, for solar zenith angle less than $40^{\circ}$, to between 14 and $17 \mathrm{~km}$ near the terminator, depending on season. Near-peak temperatures vary between $220 \mathrm{~K}$ and $300 \mathrm{~K}$, less variation than indicated by modeling, probably due to sampling near solar minimum.

Citation: Morgan, D. D., D. A. Gurnett, D. L. Kirchner, J. L. Fox, E. Nielsen, and J. J. Plaut (2008), Variation of the Martian ionospheric electron density from Mars Express radar soundings, J. Geophys. Res., 113, A09303, doi:10.1029/2008JA013313.

\section{Introduction}

[2] This paper presents a study of the variation of the Martian ionosphere, with changes in environmental conditions, as observed by the Mars Advanced Radar for Subsurface and Ionospheric Sounding (MARSIS), the radar sounder aboard the Mars Express spacecraft.

[3] Mars Express was launched on 2 June 2003 and placed in Mars orbit on 25 December 2003. The spacecraft is described with its onboard instruments by Wilson [2004], Chicarro et al. [2004], and Nielsen [2004]; the radar sounder MARSIS is described by Picardi et al. [2004]. MARSIS operates in both subsurface and ionospheric sounding modes. The subsurface mode is designed to probe below the Martian surface, a principal objective being the detection of water. Early results of the subsurface sounder are summarized by Picardi et al. [2005] with more recent subsurface results given by Plaut et al. [2007].

[4] In contrast to the subsurface mode, the ionospheric mode is designed to probe the topside ionosphere. While the subsurface mode utilizes four frequency bands, the iono-

\footnotetext{
${ }^{1}$ Department of Physics and Astronomy, University of Iowa, Iowa City, Iowa, USA.

${ }^{2}$ Department of Physics, Wright State University, Dayton, Ohio, USA.

${ }^{3}$ Max Plank Institute for Solar System Studies, Katlenburg-Lindau, Germany.

${ }^{4}$ Jet Propulsion Laboratory, Pasadena, California, USA.

Copyright 2008 by the American Geophysical Union. 0148-0227/08/2008JA013313
}

spheric mode uses only one, with frequencies between 0.1 and $5.5 \mathrm{MHz}$ corresponding to electron densities between $1.20 \times 10^{2}$ and $3.75 \times 10^{5} \mathrm{~cm}^{-3}$. These densities bracket the characteristic densities of the Martian ionosphere. Early results from the ionospheric sounder are described by Gurnett et al. [2005, 2008].

[5] Because of concerns about damage to the spacecraft, the MARSIS antenna was not deployed until 17 June 2005, over 2 years after Mars Express entered Mars orbit. Commissioning of the MARSIS ionospheric sounding mode was completed on 14 August 2005. For this study, an ending date of 1 August 2007 (orbit 4590) was chosen. Between these dates, MARSIS collected approximately 157,000 soundings, of which approximately 113,400 contain an ionospheric trace and of which 34,492 were analyzed in detail.

[6] Gurnett et al. [2005] discuss several phenomena detected by the ionospheric sounder, including oblique reflections, seen as multiple ionospheric traces, and which are found by Duru et al. [2006] and Nielsen et al. [2007b] to be related to crustal magnetic fields. Safaeinili et al. [2007] use the dispersion of the subsurface radar sounder with a fit to a Chapman layer to determine the total electron content of the nightside Martian ionosphere. They find a correlation of total electron content with the crustal magnetic fields as well as a dawn-dusk asymmetry. Nielsen et al. [2007a] compare absorption of the sounding wave in the Martian ionosphere with theoretical predictions. Morgan et al. [2006] discuss absorption of the surface reflection as an 


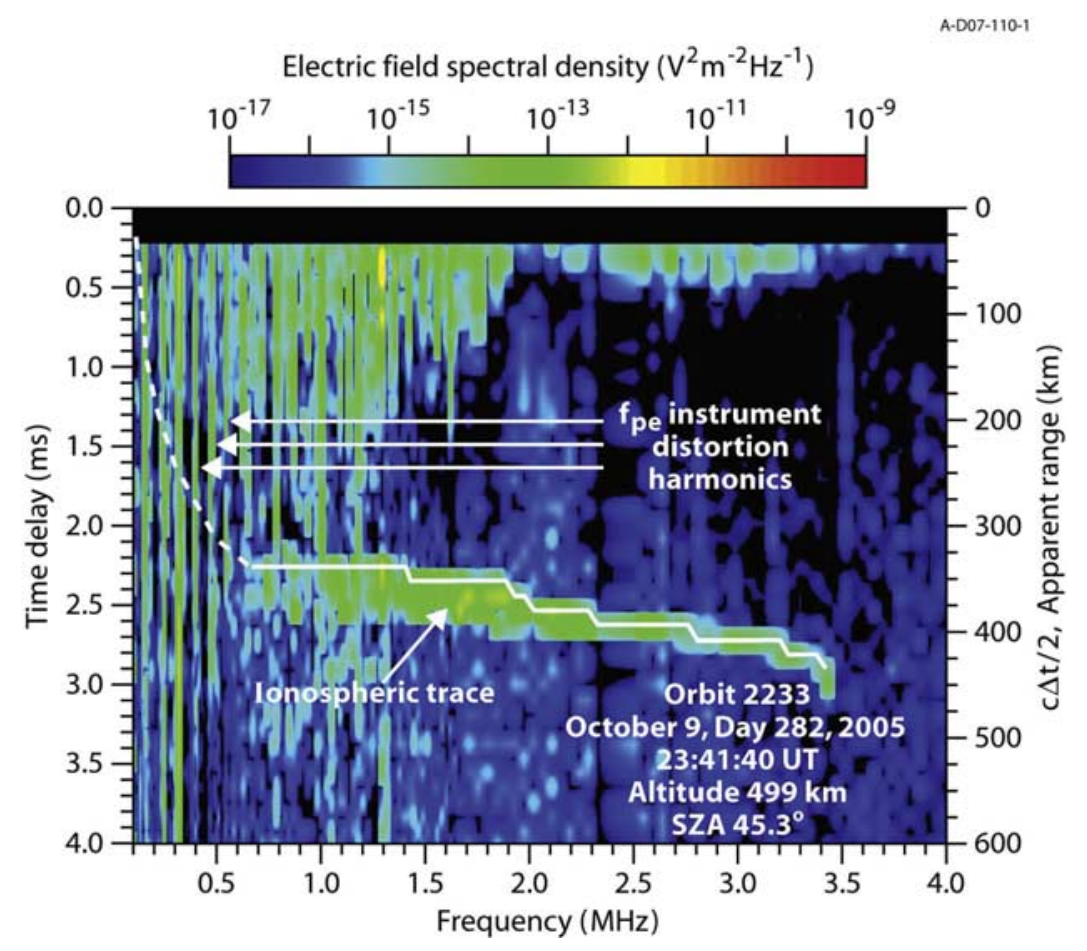

Figure 1. An ionogram: color-coded received intensity plotted as a function of sounding frequency (abscissa) and delay time (left-hand ordinate) or apparent range (right-hand ordinate). The ionospheric trace and electron plasma frequency harmonics are labeled. The solid white line shows the trace selected by the procedure given in this section. The dashed line shows where the low-frequency trace would be, based on the inversion shown in Figure 2.

indicator of the presence of solar energetic particles. This study was confirmed and extended by Espley et al. [2007] using the surface reflection from the subsurface rather than the ionospheric mode.

[7] The present study is concerned strictly with analysis of the ionospheric signal and its use in characterizing the state of the Martian ionosphere. Section 2 discusses the MARSIS instrument, its output, and the processing of that output into electron density profiles. Section 3 compares our results with radio occultation profiles from Mars Global Surveyor Radio Science and Mars Express Radio Science. In Section 4, two methods of using the Chapman ionospheric model to fit the results of our inversion technique are discussed. These methods are then used in section 5 to analyze the variation of the Martian ionosphere due to solar energetic particles and EUV flux as indexed by the solar F10.7 flux, the change of the Martian seasons (including heliocentric distance), and variation of the sampled solar zenith angle and latitude. Finally, in section 6 the variation of temperatures derived from the neutral scale heights are compared with various model results.

\section{MARSIS Ionospheric Sounding: Instrument, Data Output, and Processing}

\subsection{Instrument}

[8] The MARSIS radar sounder uses a dipole antenna consisting of two colinear $20 \mathrm{~m}$ segments for transmission of the sounding wave and for reception of its reflection. In ionospheric mode, the antenna is connected directly to the transmit/receive switch, unlike the subsurface mode, in which an impedance matching network is interposed between the antenna and the switch. The lack of a matching network in ionospheric mode results in a radiated power curve that follows the dipole resonance of the antenna, with a peak around 3.5 MHz, falling off to very low levels at $100 \mathrm{kHz}$. A dip in the radiated power around $2.5 \mathrm{MHz}$ results from a resonance between the antenna and the transmitter system output impedance.

[9] The transmit/receive switch connects the transmitter to the antenna during transmit phase and protects the receiver input from the high voltage present on the antenna. During the receive phase, the transmitter is disconnected from the antenna and connected to a low-noise preamplifier. Frequency shaping in the preamp is used to partially compensate for low radiated power levels below $1 \mathrm{MHz}$.

[10] Each sounding interval begins with the emission of a quasi-sinusoidal sounding pulse, $91.4 \mu \mathrm{s}$ in duration, at a fixed frequency. There is then a pause of $162.5 \mu$ s to protect the receiver, followed by 80 equally spaced detection intervals, each lasting $91.4 \mu \mathrm{s}$. This is followed by a pause of $278 \mu \mathrm{s}$, after which the cycle is repeated for the next frequency. This cycle is repeated for 160 frequencies between 0.1 and $5.5 \mathrm{MHz}$, giving a collection time of $1.257 \mathrm{~s}$ for one complete frequency sweep. The basic frequency sweep cycle is repeated every $7.543 \mathrm{~s}$. During each frequency sweep a $80 \times 160$ element array of received intensity as a function of delay time and sounding frequency is collected. Each array is displayed in the form of a frequency-time-delay spectrogram called an ionogram. A sample ionogram is shown in Figure 1. 


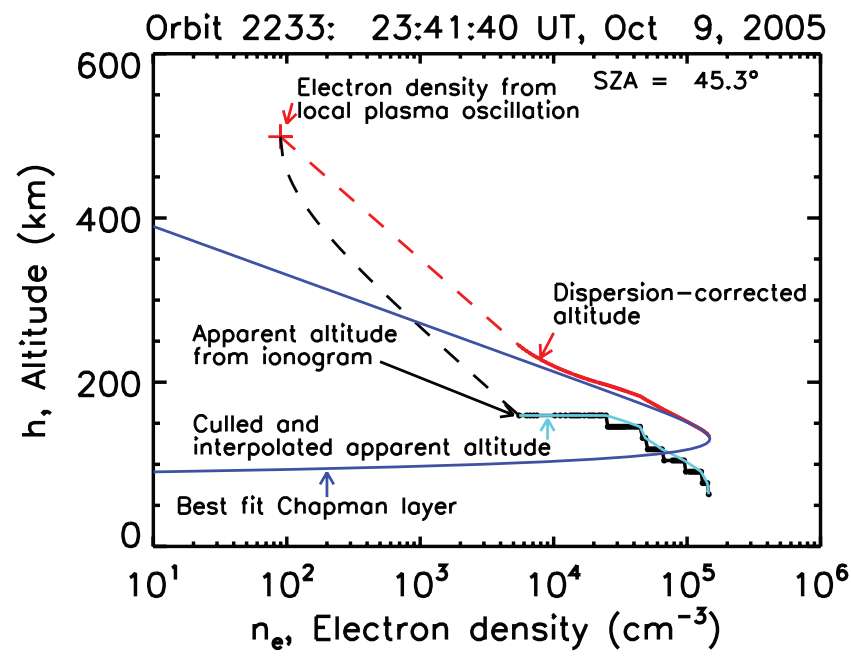

Figure 2. Processing of an ionospheric trace. The abscissa shows the plasma density corresponding to measured sounding frequency. The ordinate shows altitude, computed from either apparent or corrected range. Apparent altitude versus plasma density corresponding to the white trace in Figure 1 is shown by black dots. The blue-green line indicates the culled and interpolated apparent altitude. Red dots indicate the culled and interpolated apparent altitude corrected for dispersion according to equation (9). The blue curve shows the best fit Chapman layer for this ionospheric trace. The parameters of this Chapman layer are $n_{0}=1.76 \times$ $10^{5} \mathrm{~cm}^{-3}, h_{0}=126.6 \mathrm{~km}$, and $H=12.8 \mathrm{~km}$.

[11] Because the range at which ionospheric echoes can be detected is limited to a few thousand kilometers, each ionospheric sounding collection pass is limited to an interval of approximately $50 \mathrm{~min}$ around periapsis (between 250 and $280 \mathrm{~km}$ altitude). Each complete data collection pass generates approximately 250 ionogram arrays.

[12] An essential feature for converting an ionospheric sounding to an electron density profile is illustrated in the upper left corner of Figure 1. The bright vertical stripes represent harmonics of the plasma frequency local to the spacecraft created by the following process. The sounding wave pulse excites electron plasma oscillations at the local plasma frequency in the vicinity of the spacecraft. This oscillation couples to the antenna and drives the receiver at such high intensities that the signal is distorted and harmonics of the plasma frequency are detected in the receiver. By measuring the spacing between these harmonics, the local plasma frequency can be determined. The plasma frequency at the spacecraft, determined using these harmonics, provides the starting point for converting the delay time to corrected range. Duru et al. [2008] explain this technique and use it extensively to study the structure of the Martian ionosphere.

\subsection{Processing of Ionograms}

[13] Our analysis is initially concerned with converting the reflection of the sounding wave from the topside ionosphere, seen in Figure 1 as the mostly horizontal high-intensity trace, to a profile of electron density as a function of altitude. The ionospheric trace is converted to a numerical function of delay time versus sounding frequency by a semiautomated process in which a box is drawn around the ionospheric trace on a computer screen. The program then chooses the minimum delay-time pixel for each frequency within the box at which a selected threshold intensity, empirically set at $1 \times 10^{-15}(\mathrm{~V} / \mathrm{m})^{2} / \mathrm{Hz}$, is reached. Because the ionospheric trace is often noisy, the chosen points must be examined and those due to noise deleted. The spacecraft-local plasma frequency must also be measured. This is done by means of an on-screen adjustable scale used to determine the frequency spacing between the distortion harmonics (discussed in section 2.1 and labeled in Figure 1). These results are stored in a file for further processing. The solid white line in Figure 1 shows the result of tracing. (The dashed white curve indicates our estimate of the electron plasma frequency profile between the measured local plasma frequency and the lowest-frequency sounding point near $0.6 \mathrm{MHz}$ based on the mathematical solution to be discussed in section 2.3.)

[14] Under the assumption that the sounding wave travels at the vacuum speed of light $c$, an "apparent range," i.e., apparent distance from the spacecraft, can be calculated directly from the delay time by

$$
z_{\text {app }}=c t_{\text {delay }} / 2
$$

where $z_{\text {app }}$ is the apparent range, $t_{\text {delay }}$ the delay time, and $c$ the vacuum speed of light. Assuming that propagation is along the nadir direction, the apparent range can be converted to an apparent altitude by subtraction from the spacecraft altitude. The sounding frequencies can be converted to plasma densities by the standard relation between electron plasma frequency and electron density:

$$
n_{\mathrm{e}}\left(\mathrm{cm}^{-3}\right) \approx\left[f_{\mathrm{s}}(\mathrm{Hz}) / 8.98 \times 10^{3}\right]^{2}
$$

[see, e.g., Gurnett and Bhattacharjee, 2005, p. 11, equation (2.3.5)]. Figure 2 shows, as black dots, the ionospheric trace of Figure 1 converted to a function of apparent range versus electron density.

[15] The delay time has a resolution of $91.4 \mu \mathrm{s}$, equivalent to an uncertainty of approximately $\pm 6.8 \mathrm{~km}$ in apparent range. This resolution leads to a stairstep appearance in the ionospheric trace, as seen in the solid white trace in Figure 1. A two-step process is used to smooth the trace. The points are culled so that only upper corners of the stairstep pattern are kept. The delay times from the remaining points are then used to linearly interpolate to intermediate instrument frequencies. The interpolated values therefore represent the smallest possible delay times based on the original trace. We choose this method because the smallest possible delay time should represent a raypath that lies along the nadir direction. The result of the culling and interpolation process is shown in Figure 2 as a blue-green colored curve connecting the upper corners of the original trace. The resulting trace will be processed to create an electron density profile.

\subsection{Computation of Electron Density Profiles}

[16] The desired result from a topside sounder such as MARSIS is the electron density profile along the nadir direction. The electron density at the point of reflection is 
given directly by the sounding frequency according to equation (2); however, determination of the distance to the point of reflection is complicated by dispersion of the radio wave in regions where the plasma density and therefore the group velocity is unknown. It is therefore necessary to correct equation (1) by using the dispersion relation of an electromagnetic wave in a plasma:

$$
v_{\text {phase }}=\frac{c}{\left[1-\left(f_{\mathrm{pe}} / f_{\mathrm{s}}\right)^{2}\right]^{1 / 2}}
$$

which implies a group velocity of

$$
v_{\text {group }}=c\left[1-\left(f_{\mathrm{pe}} / f_{\mathrm{s}}\right)^{2}\right]^{1 / 2}
$$

where $v_{\text {phase }}$ is the phase velocity of the sounding wave, $f_{\mathrm{pe}}$ the plasma frequency along the ray path, $f_{\mathrm{s}}$ the sounding wave frequency, and $v_{\text {group }}$ the group velocity of the sounding wave. The unmagnetized dispersion relation is appropriate because the magnetic fields at Mars make only a small difference in the index of refraction at applicable frequencies. The time between the launching and reception of the sounding pulse, the delay time, is therefore given by

$$
t_{\text {delay }}=2 \int_{0}^{z_{\text {refl }}} \frac{d z}{v_{\text {group }}}=\frac{2}{c} \int_{0}^{z_{\text {refl }}} \frac{d z}{\left[1-\left(f_{\mathrm{pe}} / f_{\mathrm{s}}\right)^{2}\right]^{1 / 2}}
$$

where $z$ is the distance from the spacecraft along the sounding raypath and $z_{\text {refl }}$ is $z$ at the reflection point; $z_{\text {refl }}$ is called the range. Equation (5) can be applied along any ray path; however, in this application, the simplifying assumption is made that the ionosphere is horizontally stratified and that the sounding wave and its reflection travel along the spacecraft-nadir line.

[17] Note that the value of the integral is a measured quantity, whereas the value to be calculated, the range, is the upper limit of the integral. Solving this equation is referred to as inversion.

[18] The solution to equation 5 has been derived by several authors. Budden [1961] derives the equation

$$
z\left(f_{\mathrm{pe}}\right)=\frac{2}{\pi} \int_{\alpha_{0}}^{\frac{1}{2} \pi} r_{\mathrm{app}}\left(f_{\mathrm{pe}} \sin \alpha\right) d \alpha
$$

where $\alpha_{0}=\arcsin \left[\left(f_{\mathrm{pe}}(0) / f_{\mathrm{pe}}\right)\right]$ and the subscript 0 refers to the value local to the spacecraft. The theory of the solution to this equation, known as Abel's integral equation, is given by Whittaker and Watson [1927]. An alternative derivation using Laplace transformations is given by Manning [1947]. This solution works best when the data can be expressed as a continuous function.

[19] To better accommodate discrete measurements, Jackson [1969] outlines the lamination method, so called because equation (5) is solved for intervals assumed to be horizontally stratified in layers bounded by measurements. Most recently, Nielsen et al. [2006] demonstrate an inversion procedure, based on the lamination method, for ionograms from two MARSIS orbits, and show that the resulting electron density profile is well approximated by a Chapman layer. The inversion method used here, similar to that of
Nielsen et al. [2006], is also a form of the lamination method.

[20] We begin by assuming that the final result, $z$ as a function of $f_{\mathrm{pe}}$, is monotonic. The subscript $j$ is assigned to each sounding frequency and its corresponding delay time. For each $t_{\text {delay, } j}$ corresponding to sounding frequency $f_{\mathrm{s}, j}=$ $f_{\text {pe }}\left(z_{j}\right)$, we wish to compute the range $z_{j}$. In this context, the subscript 0 is reserved for values at the position of the spacecraft, so that $z_{0}=0$ and $f_{\mathrm{s}, 0}=f_{\mathrm{pe}}\left(z_{0}\right)$ is measured directly from the ionogram as described in section 2.2 . It is necessary to choose an integrable functional form that can be applied to each interval between sounding points. Jackson [1969] originally assumed a second-order logarithmic polynomial. We find a simple logarithmic form to be convenient and to give reasonable results. For each sounding frequency interval, we make the substitution

$$
z=\frac{1}{\alpha_{j}} \ln \left[\frac{f_{\mathrm{pe}}(z)}{f_{\mathrm{pe}}\left(z_{j-1}\right)}\right]=\frac{1}{\alpha_{j}} \ln \left[\frac{f_{\mathrm{pe}}(z)}{f_{\mathrm{s}, j-1}}\right]
$$

where the range of integration has been divided at the data points (designated by the subscript $j$ ),$z_{j-1}$ is the start point of the range of the $j$ th interval, and $\alpha_{j}$ is the exponential constant for the $j$ th interval. Changing variables in equation (5) from $z$ to $f_{\mathrm{pe}}$ according to equation (7) yields the time delay of the $j$ th sounding wave:

$$
t_{\text {delay }, j}=\sum_{i=1}^{i} \Delta t_{\text {delay }, i, j}=\frac{2}{c} \sum_{i=1}^{j} \int_{f_{\mathrm{s}}, i-1}^{f_{\mathrm{s}}, i} \frac{d f_{\mathrm{pe}}}{\alpha_{i} f_{\mathrm{pe}}\left[1-\left(f_{\mathrm{pe}} / f_{\mathrm{s}, j}\right)^{2}\right]^{1 / 2}}
$$

where $\Delta t_{\text {delay, } i, j}$ is the delay time for the $j$ th sounding wave frequency over the $i$ th ray path segment, and $0 \leq i \leq j$. The integrals on the right-hand side of equation (8) can now be performed by elementary methods, giving the result

$$
\begin{aligned}
t_{\text {delay }, j}= & \sum_{i=1}^{j} \Delta t_{\text {delay }, i, j}=\sum_{i=1}^{j} \frac{1}{c \alpha_{i}} \ln \left[\frac{1-\sqrt{1-\left(f_{\mathrm{s}, i} / f_{\mathrm{s}, j}\right)}}{1+\sqrt{1-\left(f_{\mathrm{s}, i} / f_{\mathrm{s}, j}\right)}}\right. \\
& \left.\cdot \frac{1+\sqrt{1-\left(f_{\mathrm{s}, i-1} / f_{\mathrm{s}, j}\right)}}{1-\sqrt{1-\left(f_{\mathrm{s}, i-1} / f_{\mathrm{s}, j}\right)}}\right]
\end{aligned}
$$

With $f_{\mathrm{s}, 0}$ given directly by the spectrogram as described in section 2.2, equation (8) constitutes a system of $j$ equations in $j$ unknowns, the $\alpha_{i}$ s. Solving for the $\alpha_{i}$ s gives a complete description of the electron density profile.

[21] Returning to Figure 2, the red curve is the apparent altitude corrected for dispersion according to equation (9). This process yields a maximum electron density $n$ (Peak) of $1.45 \times 10^{5} \mathrm{~cm}^{-3}$ at an altitude $h$ (Peak) of $134.8 \mathrm{~km}$ for this electron density profile.

\section{Comparison With Mars Express and MGS Radio Science Results}

[22] MARSIS is the first radar sounder to orbit Mars. However, previous to the launch of Mars Express, the radio occultation technique was used extensively to take both atmospheric and ionospheric density profiles. The use of this technique dates back to Mariner 4 in 1966 and includes the Viking orbiters of the 1970s; Hantsch and Bauer [1990] 


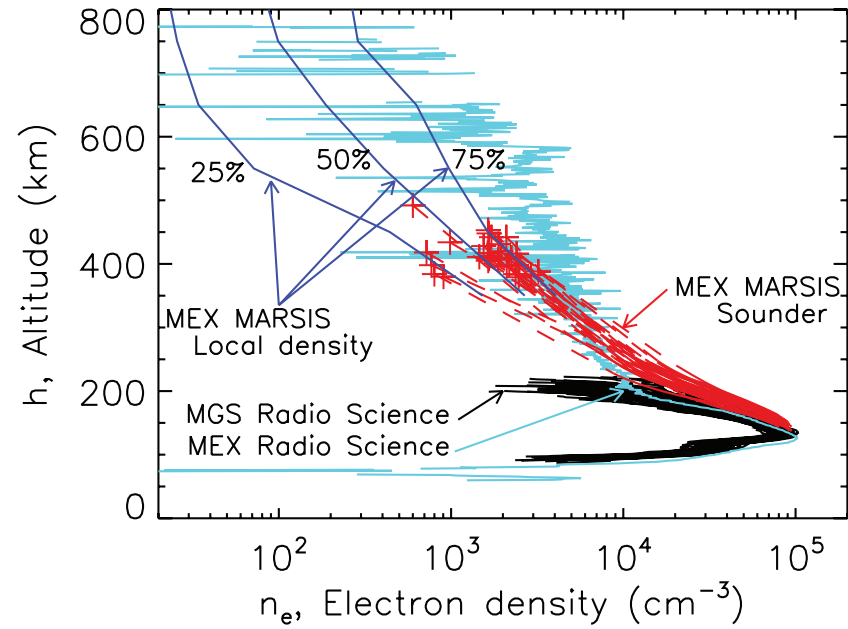

Figure 3. Comparison of MARSIS electron density profiles (red) with 46 MGS (black) and one Mars Express Radio Science (MaRS) radio occultation profile (blue-green) and with MARSIS local electron density measurements (median and upper and lower quartiles shown in blue). The MGS profiles were taken between 9 March and 27 March 1999 at solar zenith angles between $76^{\circ}$ and $78^{\circ}, L_{\mathrm{S}}$ between $107^{\circ}$ and $116^{\circ}$, and latitude between $69^{\circ}$ and $73^{\circ} \mathrm{N}$. The MaRS profile is from orbit 2464 on 13 December 2005 at solar zenith angle of $75^{\circ}, L_{\mathrm{S}}$ of $340^{\circ}$, and latitude of $65^{\circ} \mathrm{N}$. The MARSIS profiles are from orbits 2369 to 2384 on 17-21 November 2005, with solar zenith angles between $70^{\circ}$ and $80^{\circ}, L_{\mathrm{S}}$ between $326^{\circ}$ and $328^{\circ}$, and latitude between $82^{\circ}$ and $86^{\circ} \mathrm{S}$. The local electron densities are chosen for solar zenith angles between $70^{\circ}$ and $80^{\circ}$ and cover nearly the full 2 years of sampling and full ranges of $L_{\mathrm{S}}$ and latitude. That the MaRS profile is at slightly lower altitude than the MARSIS profiles for densities below $104 \mathrm{~cm}^{-3}$ is probably caused by the difference in season and hemisphere.

summarize much of this early work and Kliore [1992] compares many such profiles with similar profiles taken at Venus.

[23] Since 1998, the Mars Global Surveyor (MGS) radio occultation experiment has returned a wealth of electron density profiles using this technique. These profiles have been analyzed by Krymskii et al. [2004] and Breus et al. [2004] to show the effect of regions of intense magnetic field embedded in the planetary crust on the Martian ionosphere. Rishbeth and Mendillo [2004], Martinis et al. [2003], Withers and Mendillo [2005], and Wang and Nielsen [2003a] have used radio occultation electron density profiles to analyze diurnal variations and possible wave effects on the Martian ionosphere and to compare with diurnal variations at Earth. Modeling efforts based on MGS radio occultation include those of Bougher et al. [2000] and Fox and Yeager [2006]. More recently, Pätzold et al. [2005] have used the radio occultation technique from Mars Express to detect an intermittent ionospheric layer below the principal ionospheric maximum, attributed to the effect of meteor ablations [Pesnell and Grebowsky, 2000].

[24] The radio occultation technique utilizes the dispersion of radio waves passing through any dispersive medium to gauge the density of that medium. Hinson et al. [1999], who give a detailed explanation of radio occultation, use the radio signals passed between Mars Global Surveyor and receiving stations on Earth to measure Martian neutral atmosphere densities. The primary advantage of radio occultation over radar sounding is that it can probe below density maxima where the ionospheric plasma is shielded from a topside sounding wave. The primary disadvantage of radio occultation with respect to radar sounding is that occultation geometry limits the sampling to solar zenith angles greater than $\sim 45^{\circ}$.

[25] In Figure 3, we compare MGS and Mars Express Radio Science (MaRS) radio occultation profiles with MARSIS electron density profiles taken between $70^{\circ}$ and $80^{\circ}$ solar zenith angle. The 46 MGS profiles, shown in black, were taken between 9 March and 27 March 1999 at solar zenith angles of $76^{\circ}$ to $78^{\circ}$, with solar longitudes between $107^{\circ}$ and $116^{\circ}$, and latitudes between $69^{\circ}$ and $73^{\circ}$. Some of these profiles have been published by Rishbeth and Mendillo [2004] and Martinis et al. [2003]. A single MaRS profile, shown as a blue-green curve, was taken on 13 December 2005 on Mars Express orbit 2464. The solar zenith angle for this profile is $75^{\circ}$, solar longitude is $340^{\circ}$, and latitude is $65^{\circ} \mathrm{N}$. The MARSIS profiles for comparison are shown in red and were taken from 7 November to 21 December 2005 during orbits 2369 through 2384 at solar zenith angles between $70^{\circ}$ and $80^{\circ}$, solar longitudes between $326^{\circ}$ and $328^{\circ}$, and latitudes between $82^{\circ}$ and $86^{\circ} \mathrm{S}$. The large plus signs mark the local density and altitude at the position of the Mars Express spacecraft for each MARSIS profile and the dashed red lines indicate the region between the spacecraft and the first sounding point.

[26] From the density peak at about $130 \mathrm{~km}$ up to about $200 \mathrm{~km}$ altitude and electron densities greater than about $10^{4} \mathrm{~cm}^{-3}$, the three profiles are in reasonable agreement, with the MARSIS profiles appearing to be slightly higher in altitude than either MGS or MaRS. This difference is probably due to the difference in hemisphere coupled with season: the MGS profiles were taken in northern summer near perigee, while the MaRS profile was taken in northern winter near apogee, and the MARSIS profiles were taken in southern summer, also near apogee. As will be seen, profile altitudes tend to be elevated during summer as the poles are approached.

[27] The MGS profiles are truncated above about $200 \mathrm{~km}$ altitude so that it is difficult to make comparisons with the other profiles; however, they appear to give lower densities than the other two sets as the altitude increases. In contrast, the MaRS profile appears to give higher densities as the altitude increases from this point, although the precision of the occultation result appears to fall off rapidly at higher altitudes. The MARSIS profiles fall between the two sets of occultation profiles. However, the dashed lines indicate that most of the data in this range is the result of integral interpolation between the local spacecraft density and the first sounding point, indicating a dearth of sounding at these altitudes. All three sets of profiles and both methods suffer from a lack of precision above $200 \mathrm{~km}$.

[28] For further comparison, we have also shown the results of MARSIS local density measurements, at solar zenith angles between $75^{\circ}$ to $80^{\circ}$, taken over a much longer 
period, and covering nearly the full range of latitude and $L_{\mathrm{S}}$. The local densities are shown by blue lines, representing the median and upper and lower quartiles of the density as a function of altitude. The techniques for measuring and analyzing the local plasma density are discussed in detail by Duru et al. [2008]. The MARSIS profiles are consistent with the MARSIS local electron density measurements above altitudes of $200 \mathrm{~km}$ and densities less than $1 \times 10^{3} \mathrm{~cm}^{-3}$. This is not surprising, since the MARSIS profiles are in part based on the MARSIS local electron density measurements.

[29] We conclude that the sounding and radio occultation methods are in approximate agreement in the near-peak region below $200 \mathrm{~km}$ altitude for solar zenith angles between $75^{\circ}$ and $80^{\circ}$. The differences can probably be explained as the result of sampling different hemispheres. At higher altitudes, the three sets of profiles give divergent results; however, the MARSIS profiles are supported by the MARSIS local electron density measurements. The MaRS profile shows signs of a layer below the main peak. Pätzold et al. [2005] claim that similar layers are due to meteor ablation [see Pesnell and Grebowsky, 2000]. However, this process occurs below the principal ionospheric peak and probably does not affect electron densities at MARSIS sounding altitudes.

\section{Fitting Electron Density Profiles to the Chapman Ionospheric Model}

[30] In order to compare electron density profiles sampled at varying values of the solar zenith angle, it is necessary to adopt an ionospheric model. The simplest such model is the Chapman model, derived by Sidney Chapman in two papers in 1931 [Chapman, 1931a, 1931b; see also Rishbeth and Garriott, 1969; Schunk and Nagy, 2000]. The Chapman model assumes monochromatic ionizing radiation, a single ionizable species, an isothermal neutral atmosphere, and photochemical equilibrium. Conditions in the Martian ionosphere differ from these assumptions in a number of ways: the Martian ionosphere is created by a fairly wide range of photons, from 150 to 1000 Angstroms [Fox and Yeager, 2006]; the temperature profile shown by Krasnopolsky [2002] is nonisothermal at the altitudes with which we are primarily concerned (between 100 and $180 \mathrm{~km}$ ); the Martian ionosphere is not in photochemical equilibrium above about $180 \mathrm{~km}$ altitude [Fox and Yeager, 2006]. On the other hand, one ionizable species, $\mathrm{CO}_{2}$, makes up $92 \%$ of the neutral atmosphere (although above $200 \mathrm{~km} \mathrm{O}$ is the most important neutral species). Furthermore, in spite of the temperature gradient, the density profile of $\mathrm{CO}_{2}$ is approximately exponential in the altitude range of interest [Krasnopolsky, 2002]. Finally, photochemical equilibrium is thought to be a good approximation for the Martian atmosphere near the electron density maximum [Bougher et al., 2001; Schunk and Nagy, 2000, p. 443] and was found to be so by Withers and Mendillo [2005] and Nielsen et al. [2006] (although Zou et al. [2006] find small deviations from Chapman theory, with the sense of deviation depending on hemisphere). We will therefore use the Chapman model to organize our results by referring the values of $n(P e a k)$ and $h($ Peak) to the subsolar point and making our results roughly comparable on a global basis.
[31] The electron density profile of a Chapman layer is given by the equation [Chapman, 1931a, 1931b]

$$
n_{\mathrm{e}}=n_{0} \exp \left\{\frac{1}{2}\left[1-\frac{h-h_{0}}{H}-\operatorname{Ch}(\chi, x) \exp \left(-\frac{h-h_{0}}{H}\right)\right]\right\}
$$

where $n_{\mathrm{e}}$ is the electron density, $h$ is the altitude, $H$ is the neutral scale height of the planetary atmosphere, $\chi$ is the solar zenith angle, $n_{0}$ is the subsolar maximum electron density, $h_{0}$ is the altitude of the subsolar maximum electron density, and $\operatorname{Ch}(\chi, x)$ is the Chapman grazing incidence function. Here $x=(h+R) / H$ and $R=3396.19 \mathrm{~km}$ is the radius of Mars. An approximation to this function given by Smith and Smith [1972] is used. Equation (10) has three free parameters, the subsolar maximum electron density $n_{0}$, the altitude of the subsolar peak electron density altitude $h_{0}$, and the atmospheric neutral scale height $H$.

\subsection{Fitting Individual Electron Density Profiles to a Chapman Layer}

[32] In order to fit an electron density profile, such as that shown in red in Figure 2, to equation (10), the errors on the sounding frequencies are estimated to be equivalent to half the bandwidth of the receiver, $\sim 5 \mathrm{kHz}$. The density errors can be computed by $d n_{e} / n_{e}=2 \times d f_{\text {pe }} / f_{\text {pe }}$. Using a standard least squares curve-fitting routine, the reduced chi-square $\chi_{v}^{2}$ is computed for the set of all points in the inverted curve. Then $\chi_{v}^{2}$ is recomputed for all except the lowest-frequency point. The next lowest-frequency point is then removed and $\chi_{v}^{2}$ recomputed, continuing until there are only six points (three degrees of freedom). The fit that gives the minimum $\chi_{v}^{2}$ is chosen as the answer. Our procedure is chosen to preferentially select fits at altitudes near the electron density maximum, since this is where the ionosphere is most likely to be in photochemical equilibrium while the $\mathrm{CO}_{2}$ density profile is fairly well approximated by a constant scale height. The blue curve in Figure 2 is the Chapman layer derived by this procedure for the ionospheric trace shown in Figure 1. In agreement with our methodology, the fit agrees closely with the inversion result near the plasma density peak and differs significantly at higher altitudes. For this case, the parameters for the inferred Chapman layer are $n_{0}=$ $1.76 \times 10^{5} \mathrm{~cm}^{-3}, h_{0}=126.6 \mathrm{~km}$, and $H=12.8 \mathrm{~km}$.

\subsection{Fitting Accumulated Inversion Results to a Chapman Layer}

[33] The Chapman grazing incidence function $\operatorname{Ch}(\chi, x)$ accounts for deviation from the approximation of plane parallel geometry, which occurs at values of $\chi$ approaching $90^{\circ}$. For reasonable values of the neutral scale height $(\sim 10$ $\mathrm{km}$ at Mars), plane parallel geometry is a good approximation for $\chi<85^{\circ}$. In this case, $\operatorname{Ch}(\chi, x) \approx \sec (\chi)$, and the maximum value of $n_{\mathrm{e}}$ with its altitude can be found by differentiating equation (10) and solving for the maximum of $n_{\mathrm{e}}$ with respect to $h$. The resulting equations are given, for example, in the work of Hantsch and Bauer [1990], as

$$
n_{\mathrm{e}}(\text { Peak }) \approx \mathrm{n}_{0} \cos ^{1 / 2} \chi
$$

and

$$
h(\text { Peak }) \approx h_{0}-H \times \ln (\cos \chi)
$$



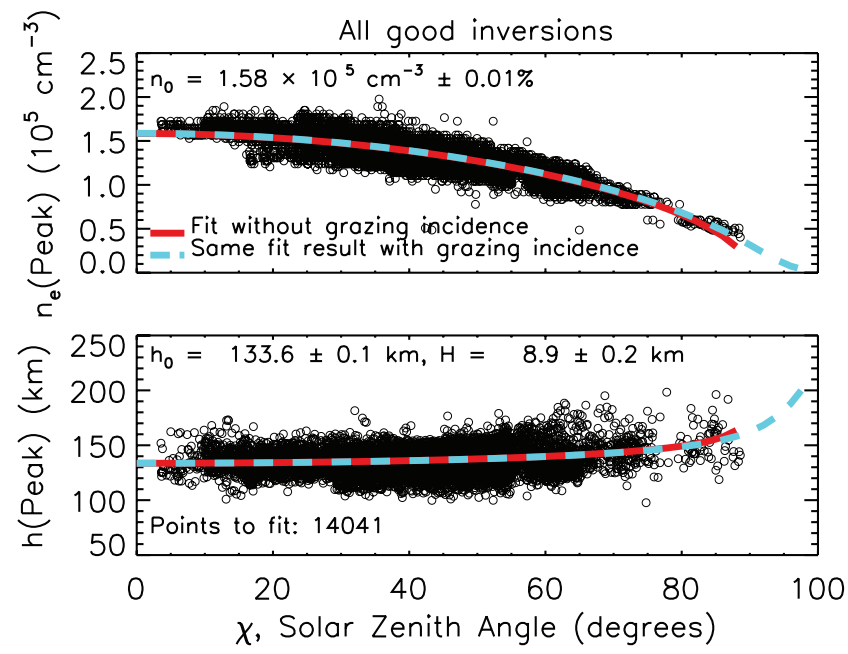

Figure 4. Plots of (top) $n_{\mathrm{e}}$ (Peak) and (bottom) $h$ (Peak) for good inversions (as explained in section 4.3) against solar zenith angle $\chi$. Only points for which $\chi<85^{\circ}$ are used for fitting. The red curves are the result of the fits done separately for the top and bottom. The dashed blue-green curves show the effect of using these values in equation (10) with grazing incidence included.

where $n_{\mathrm{e}}$ (Peak) is the maximum value of the electron density calculated for a given profile from the maximum plasma frequency by equation (2), and $h$ (Peak) is the altitude of the density peak calculated for a given profile from equation (9). Thus, we can plot the accumulated values of $n_{e}$ (Peak) and $h$ (Peak) as a function of the solar zenith angle $\chi$ and fit the resulting distribution to equations (11) and (12) for the three free parameters, $n_{0}, h_{0}$, and $H$ defined above. When this method is used, points for which $\chi>85^{\circ}$ are excluded. The uncertainty on $n_{\mathrm{e}}$ (Peak) is as in section 4.1. The uncertainty on $h$ (Peak) is shown by direct calculation to be reasonably well approximated by resolution of the apparent range, given by $91.4 \mu \mathrm{s} \times c / 2 \times 1 / 2 \approx$ $6.8 \mathrm{~km}$.

\subsection{Selection of Traces for Fitting Procedures}

[34] Not all of the ionospheric traces accumulated are adequate for either inverting or fitting to a Chapman layer. The traces are therefore culled to select those that are reasonably complete and are not likely to include a ledge or nonmonotonic segment in the electron density profile. Inverted profiles that are patently unphysical are also eliminated. To this end, the following conditions on our data set are imposed. (1) The ionospheric trace must be at least $1 \mathrm{MHz}$ in extent in frequency. (2) The maximum allowed gap in the detected trace is $0.4 \mathrm{MHz}$. (3) The maximum allowed jump in delay time is $0.2 \mathrm{~ms}$. (4) The trace must end in a discernible "hook," indicated by the second derivative $d^{2} t_{\text {delay }} / d f^{2}>3 \mathrm{~ms} / \mathrm{MHz}^{2}$. (5) The trace must not end at frequency between 2.2 and $2.5 \mathrm{MHz}$. (6) The spacecraft altitude must be less than $800 \mathrm{~km}$. (7) The corrected altitude as a function of electron density must be monotonic.

[35] Condition 1 is imposed to ensure an adequate trace to invert; condition 2 is imposed to eliminate large frequency gaps in the data; condition 3 is imposed to eliminate cases where there is a large "cusp," indicating a ledge or overhang where the electron density profile is nonmonotonic; condition 4 is imposed to make sure that the trace closely approaches the maximum plasma frequency (since the hook at the end of the trace is important in constraining individual profile fits); condition 5 is imposed to eliminate traces that end in the sensitivity gap between receivers near $2.4 \mathrm{MHz}$ as these traces are likely to be incomplete; condition 6 is imposed to minimize possible bias due to the inversion process having to integrate over very large distances between data points; and condition 7 is imposed to eliminate cases where the altitude decreases and then increases, giving a multiple value for the density at a given point (which can occasionally happen when the trace is sporadic or noisy).

[36] We henceforth refer to the set of traces that meet these criteria as "good inversions," or as being wellinverted. Of the 34,492 traces attempted between orbits 2031 on 14 August 2005 and 4590 on 31 July 2007, 14,060 meet the criteria for being well-inverted. The wellinverted profiles are then fit to the Chapman model, as explained in section 4.1. For statistical use, these fits must meet the criterion that the three output parameters must have less than 3\% uncertainty. Of 14,060 well-inverted profiles, 12,291 meet the criterion for being well fit.

[37] As an example of fitting equations (11) and (12), Figure 4 displays the result of plotting $n_{\mathrm{e}}$ (Peak) and $h$ (Peak) for all of the aforementioned good inversions against solar zenith angle $\chi$. The plots are then fit to equations (11) and (12). The top panel of (Figure 4) shows $n_{\mathrm{e}}$ (Peak) plotted against solar zenith angle, which is similar to Figure $3 \mathrm{~b}$ of Gurnett et al. [2005]. The standard deviation of the input electron densities is estimated to be about $1.23 \times 10^{4} \mathrm{~cm}^{-3}$ or $8 \%$ of the actual value, a fairly large variation. The red curve shows the result of fitting equation (11) for $\chi \leq 85^{\circ}$. The result of the fit is $n_{0}=1.58 \times 10^{5} \mathrm{~cm}^{-3}$.

[38] The bottom panel of Figure 4 shows $h$ (Peak) against solar zenith angle. The red curve shows the result of fitting equation (12) to these data, again for $\chi \leq 85^{\circ}$. The standard deviation of the input peak altitudes is estimated to be $9.2 \mathrm{~km}$ or $7 \%$ of the value of the peak altitude, again a large variation. The results are $h_{0}=133.6 \mathrm{~km}$ and $H=8.9 \mathrm{~km}$. For comparison, averages of the Chapman parameters from individual fits are taken giving $n_{0}=1.63 \times 10^{5} \mathrm{~cm}^{-3}, h_{0}=$ $128.2 \mathrm{~km}$ and $H=12.9 \mathrm{~km}$. Standard deviations for individual fit averages are $\sigma_{n 0}=1.55 \times 10^{4} \mathrm{~cm}^{-3}, \sigma_{h 0}=$ $11.1 \mathrm{~km}, \sigma_{H}=2.7 \mathrm{~km}$.

[39] Since all three free parameters of equation (10) for the density profile of a Chapman layer are now available, $n_{\mathrm{e}}($ Peak) and $h$ (Peak) as functions of solar zenith angle with grazing incidence included are calculable. These results are shown by the dashed blue-green curves in both panels of Figure 4 . The inclusion of grazing incidence makes a visible improvement in the fit of $n_{\mathrm{e}}$ (Peak) at $\chi>85^{\circ}$; however, because of the variability of $h$ (Peak) at high solar zenith angle, it is difficult to say whether grazing incidence brings about an improvement.

[40] The values of $\chi_{v}^{2}$ for the two fits in Figure 4 are 36.3 and 2.1, indicating that the fits are not statistically good. This is as expected, since the sample spans nearly two years and includes a wide range of environmental conditions, and the input data have standard deviations approaching $10 \%$. 
Table 1. Summary of Near-Peak Temperatures From Derived Neutral Scale Heights ${ }^{\mathrm{a}}$

\begin{tabular}{|c|c|c|c|c|}
\hline$L_{\mathrm{S}}(\mathrm{deg})$ & $-68-22^{\mathrm{b}}(\mathrm{deg})$ & $22-112^{\mathrm{c}}(\mathrm{deg})$ & $112-202^{\mathrm{d}}(\mathrm{deg})$ & $202-292^{\mathrm{e}}(\mathrm{deg})$ \\
\hline \multicolumn{5}{|l|}{ SZA (deg) } \\
\hline \multicolumn{5}{|c|}{ Northern Hemisphere: $65<$ F10. $7_{\text {Earth }} \leq 80$} \\
\hline $0-30$ & $231.1 \pm 10.6$ & $231.1 \pm 3.5$ & - & - \\
\hline $30-60$ & $249.8 \pm 6.3$ & $234.8 \pm 1.0$ & - & $276.5 \pm 5.2$ \\
\hline \multirow{2}{*}{\multicolumn{5}{|c|}{ Northern Hemisphere: $85<$ F10.7 $7_{\text {Earth }} \leq 105$}} \\
\hline & & & & \\
\hline $0-30$ & - & - & - & - \\
\hline $30-60$ & - & $229.2 \pm 5.4$ & - & - \\
\hline $60-90$ & - & - & - & - \\
\hline \multicolumn{5}{|c|}{ Southern Hemisphere: $65<$ F10.7 $7_{\text {Earth }} \leq 85$} \\
\hline $0-30$ & $220.7 \pm 1.7$ & - & - & $228.6 \pm 1.3$ \\
\hline $30-60$ & $226.8 \pm 2.2$ & $257.3 \pm 4.8$ & - & $242.1 \pm 0.7$ \\
\hline $60-90$ & $271.2 \pm 6.5$ & $266.9 \pm 7.2$ & - & $302.1 \pm 6.8$ \\
\hline \multicolumn{5}{|c|}{ Southern Hemisphere: $85<$ F10.7 $7_{\text {Earth }} \leq 105$} \\
\hline $0-30$ & $244.3 \pm 6.6$ & - & - & - \\
\hline $30-60$ & $243.4 \pm 2.9$ & - & - & - \\
\hline $60-90$ & $269.5 \pm 4.1$ & - & - & - \\
\hline
\end{tabular}

${ }^{\mathrm{a}}$ Temperatures in degrees kelvin.

${ }^{\mathrm{b}}$ Northern hemisphere spring equinox, median $R=1.50 \mathrm{AU}$.

${ }^{\mathrm{c}}$ Northern hemisphere summer solstice, median $R=1.65 \mathrm{AU}$.

${ }^{\mathrm{d}}$ Southern hemisphere spring equinox, median $R=1.51 \mathrm{AU}$.

${ }^{\mathrm{e}}$ Southern hemisphere summer solstice, median $R=1.39 \mathrm{AU}$.

We will explore the effects of some of these conditions in following sections.

\section{Statistical Results}

[41] In this section, the fitting methods discussed in section 4 are used, with accumulated electron density profiles, to characterize variation of the Martian ionosphere with changes in several variables. First, we shall look at the effect of solar energetic particles on the Chapman layer parameters. We shall then analyze the ionospheric characteristics as a function of the Martian seasons (including heliocentric distance) in conjunction with solar EUV flux, solar zenith angle, and latitude.

\subsection{Effect of Solar Energetic Particles}

[42] Morgan et al. [2006] and Espley et al. [2007] detected changes in the absorption of the ionospheric and subsurface sounding waves due to excess ionization caused by solar energetic particles (SEPs). Particle events were detected through background detection of the MGS Electron Reflectometer and the Odyssey Gamma Ray Spectrometer. Table 1 of Morgan et al. [2006] gives start and stop times of known SEP events at Mars.

[43] Espley et al. [2007], found no particle events affecting the Martian ionosphere between the end of the SEP events tabulated by Morgan et al. [2006] on 23 September 2005 and 3 December 2005. The earliest good inversions in our data set are for 14 August 2005. Therefore, the period between 14 August and 3 December 2005 is taken as the sampling period for solar energetic particle events. Figures 5 and 6 show the results of plotting $n_{\mathrm{e}}$ (Peak) and $h$ (Preak) as a function of solar zenith angle for times when there is no SEP event and when an SEP event is in progress. In both cases, equations (11) and (12) are used to fit the results. The standard deviation of the input densities is estimated at $7.4 \times 10^{3} \mathrm{~cm}^{-3}$ for both SEP and non-SEP periods. The standard deviation of the input peak altitudes is estimated at
$6.7 \mathrm{~km}$ for SEP and $6.3 \mathrm{~km}$ for non-SEP periods. The red and blue-green curves have the same meaning as in Figure 4.

[44] The result of this procedure is as follows: the presence of solar energetic particles is associated with an increase in $n_{0}$ from $1.65 \times 10^{5}$ to $1.74 \times 10^{5} \mathrm{~cm}^{-3}$, an increase in $h_{0}$ from 130.2 to $134.3 \mathrm{~km}$, and a decrease in $H$ from 15.4 to $8.2 \mathrm{~km}$. The ionization has increased by about $6 \%$, the peak altitude has increased by $3 \mathrm{~km}$, and the neutral scale height has decreased by $7 \mathrm{~km}$. The reasons for the increase in peak altitude and decrease in scale height are obscure but probably have to do with particle penetration depth as a function of the SEP energy spectrum at Mars and
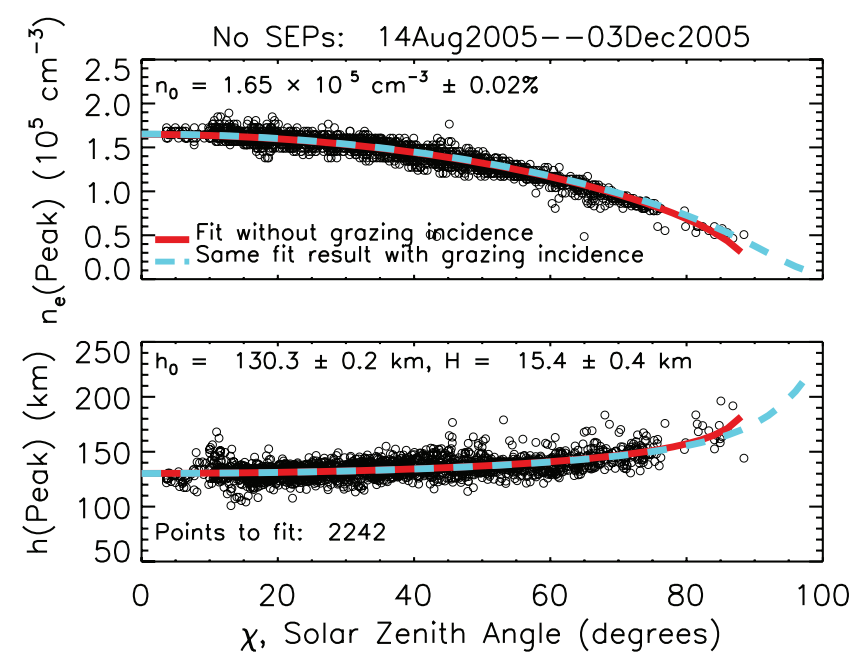

Figure 5. Fits to equations (11) and (12) for well-inverted traces occurring between 27 August and 3 December 2005 during which no SEP events were observed. (top) Fit of $n_{\mathrm{e}}($ Peak) as a function of solar zenith angle to equation (11) yielding $n_{0}=1.65 \times 10^{5} \mathrm{~cm}^{-3}$. (bottom) Fit of $h($ Peak) as a function of solar zenith angle to equation (12), yielding $h_{0}=$ $130.3 \mathrm{~km}$ and $H=15.4 \mathrm{~km}$. 

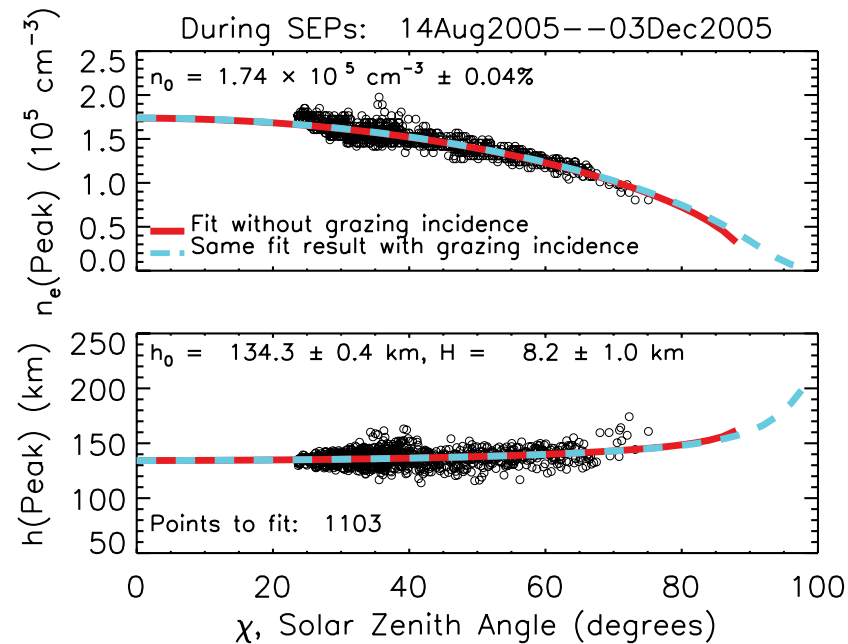

Figure 6. Fits to equations (11) and (12) for well-inverted traces occurring between 14 August and 3 December 2005 during which SEP events were recorded by Morgan et al. [2006] and Espley et al. [2007]. (top) Fit of $n_{\mathrm{e}}($ Peak) as a function of solar zenith angle to equation (11), yielding $n_{0}=$ $1.74 \times 10^{5} \mathrm{~cm}^{-3}$. (bottom) Fit of $h($ Peak) as a function of solar zenith angle to equation (12), yielding $h_{0}=134.3 \mathrm{~km}$ and $H=8.2 \mathrm{~km}$.

the detailed chemistry of the Martian neutral atmosphere (see Schröter et al. [2006] for a discussion of SEP ionization profiles at Earth).

[45] For comparison, we have averaged results of individual good fits (as in Figure 2), as defined in section 4.1, for times with and without SEP events. Using this method, the presence of solar energetic particles is associated with an increase in $n_{0}$ from $1.65 \times 10^{5}$ to $1.76 \times 10^{5} \mathrm{~cm}^{-3}$, an increase in $h_{0}$ from 127.2 to $130.1 \mathrm{~km}$, with $H$ remaining nearly constant at 12.6 to $12.4 \mathrm{~km}$. These are equivalent to an increase in $n_{0}$ of $6 \%$, an increase in $h_{0}$ of $3 \mathrm{~km}$, and a negligible change in $H$. The changes in $n_{0}$ and $h_{0}$ are consistent for the two methods, but for $H$ the two methods give divergent results. This discrepancy may be a sampling effect due to lack of range in solar zenith angle when using equations (11) and (12), especially during solar energetic particle events. Many trials have shown that the two fitting methods agree reasonably well for computation of $n_{0}$ and $h_{0}$ but that there can be significant discrepancies in the results for $H$.

\subsection{Seasonal Variation}

[46] In Figure 7, we organize the results of individual fits by Mars solar longitude $L_{\mathrm{S}}$. This quantity represents the orbital angle between the current position of Mars and the Martian northern hemisphere vernal equinox. Thus, $L_{\mathrm{S}}=$ $90^{\circ}$ represents summer solstice, $L_{\mathrm{S}}=180^{\circ}$ the autumnal equinox, and $L_{\mathrm{S}}=270^{\circ}$ the winter solstice, all in the northern hemisphere. We have ordered the results of individual good fits by $L_{\mathrm{S}}$ and put them in bins of about 100 for averaging. Shown in Figures $7 \mathrm{a}-7 \mathrm{c}$ are the average values of the derived Chapman parameters: the subsolar peak electron density $n_{0}$, the altitude of the subsolar peak electron density $h_{0}$, and the neutral atmosphere scale height $H$. In Figures $7 \mathrm{a}-7 \mathrm{c}$, the points are represented by both vertical and horizontal bars. The horizontal bars represent the extent of the data in a given bin in the organizing parameter, in this case $L_{\mathrm{S}}$. The vertical bar (often too small to see) represents the error on the mean of the bin-averaged value of the parameter. For the three derived Chapman parameters, this scheme will be followed for all subsequent analysis.

[47] There are three well-defined periods of heavy data collection, color coded as follows: 14 August 2005 to 31 January 2006 (color coded blue), 31 January 2006 to 16 February 2007 (color coded blue-green), and 16 February 2007 to 1 August 2007 (color coded red). In all subsequent analysis, this color coding is maintained.

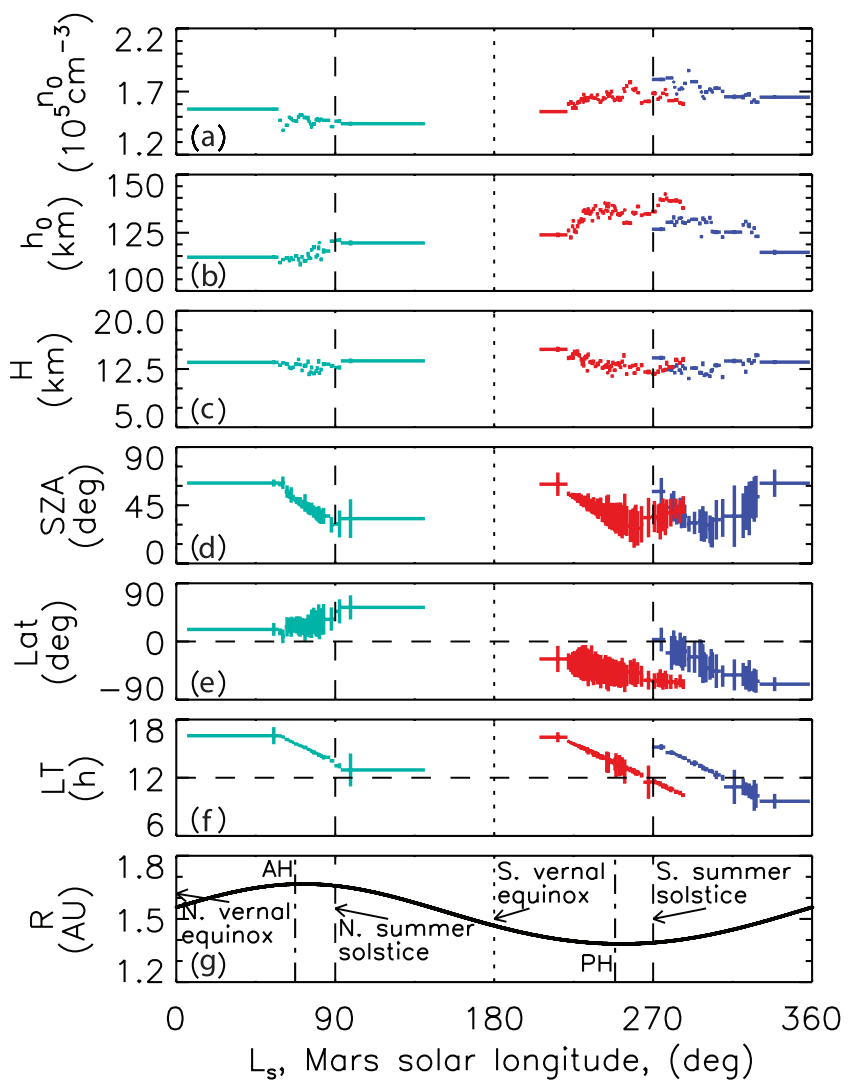

Figure 7. The Chapman layer fit parameters and three orbit parameters sorted by Mars solar longitude $L_{\mathrm{S}}$ and averaged in bins of $\sim 100$, with the heliocentric distance of Mars as a function of $L_{\mathrm{S}}$. Horizontal bars, where visible, give the range of each bin in $L_{\mathrm{S}}$. $(\mathrm{a}-\mathrm{c})$ Vertical bars, where visible, give the bin error on the mean. $(\mathrm{d}-\mathrm{f})$ Vertical bars give the standard deviation of the binned parameters, to indicate the spread in the sampling of these parameters. Colors blue, blue-green, and red stand for data Intervals 1, 2 , and 3 as given in the text. Figure 7a shows subsolar peak electron density $n_{0}$. Figure $7 \mathrm{~b}$ shows subsolar electron density peak altitude $h_{0}$. Figure $7 \mathrm{c}$ shows neutral scale height $H$. Figure $7 \mathrm{~d}$ shows solar zenith angle. Figure $7 \mathrm{e}$ shows latitude. Figure $7 \mathrm{f}$ shows local time. Figure $7 \mathrm{~g}$ shows Mars heliocentric distance $R$, with equinoxes, solstices, aphelion (AH), and perihelion ( $\mathrm{PH})$ values labeled. Parameters $n_{0}$ and $h_{0}$ are seen to vary coherently with season and consequently with Mars heliocentric distance. A seasonal variation of $H$ is not seen. 
[48] Figures 7d-7f, bin-average values of the solar zenith angle, latitude, and local time are presented. As in Figures $7 \mathrm{a}-7 \mathrm{c}$, the horizontal bar represents the extent of each bin in the organizing parameter; however, the vertical bar here represents the standard deviation of the binned orbital parameter. We present the data this way to give an idea of the orbital biases that might affect any conclusions to be drawn.

[49] In Figure $7 \mathrm{~g}$, the Martian heliocentric distance is presented as a function of $L_{S}$. Here, the seasonal attributes of the values of $L_{\mathrm{S}}$ are labeled, with solstices as dashed lines and Martian aphelion (1.67 AU) and perihelion (1.38 AU) labeled at $67^{\circ}$ and $247^{\circ}$. Because of the eccentricity of the Martian orbit, northern hemisphere winter and southern hemisphere summer will be subject to higher insolation than corresponding seasons in opposite hemispheres.

[50] Figures $7 \mathrm{f}-7 \mathrm{~g}$ show that data Interval 1, in blue, is almost completely in the southern hemisphere in summer, Interval 2, in blue-green, is in the northern hemisphere in spring and summer, and Interval 3, in red, is in the southern hemisphere in spring and summer. Zou et al. [2005] have demonstrated seasonal variation in $h_{0}$ based on MGS radio occultation profiles taken over a highly restricted range in solar longitude for northern and southern hemispheres in opposite seasons. As shown in Figures $7 f-7 g$, our data set almost completely consists of spring and summer samples; those samples that occur in winter are very near the equator. Therefore, we cannot make an effective comparison with the results of Zou et al. [2005].

[51] Figure 7a shows that the average value of $n_{0}$ varies between $1.4 \times 10^{5} \mathrm{~cm}^{-3}$ near the northern hemisphere summer solstice to $1.8 \times 10^{5} \mathrm{~cm}^{-3}$ near the southern hemisphere summer solstice. This binning of the data also reveals smaller scale variations that are probably related to the sampling distributions of solar zenith angle, latitude, and local time.

[52] The average value of $h_{0}$ varies from about $110 \mathrm{~km}$ near $L_{\mathrm{S}}=90^{\circ}$, to as high as $140 \mathrm{~km}$ at $L_{\mathrm{S}}=180^{\circ}$. The average value of $H$ is approximately constant between 12 and $15 \mathrm{~km}$ through the seasons, with some variation that is probably attributable to the orbit of Mars Express.

[53] Thus, in this plot gross differences between northern and southern hemisphere spring and summer values of $n_{0}$ and $h_{0}$ are evident. The values of the neutral scale height $H$ do not show a strong dependence on season. While the seasonal variation of $n_{0}$ and $h_{0}$ agree with previous results [see Bougher et al., 2000, 2001; Breus et al., 2004], the lack of seasonal variation of the neutral scale height does not. We shall see in sections 5.4 and 6 that there is some seasonal variation in $H$ that is partially masked by our averaging procedure.

\subsection{Effect of Variation of EUV Flux}

[54] The flux of the solar 10.7-cm radio emission, sampled from Earth orbit and given in units of $10^{-22} \mathrm{~W} \mathrm{~Hz}^{-1} \mathrm{~m}^{-2}$, is called F10.7 [see, e. g., Schunk and Nagy, 2000, p. 242]. This quantity is widely used as an index of solar activity and the intensity of solar extreme ultraviolet (EUV) flux that causes the main ionization peak near $130 \mathrm{~km}$ altitude. The day-averaged values of F10.7 are posted on the NOAA Sun-Earth Monitor Web site.
[55] Because F10.7 is sampled from geosynchronous Earth orbit, it is necessary to correct it for use as a proxy for EUV flux at Mars. Two corrections are needed: for relative orbital position and for distance from the Sun. The first correction is possible because the first-order variation of F10.7 is due to the solar rotation, which has a period of approximately 27 days. To compensate for our sampling of solar radiation at the azimuth of Mars rather than that of Earth, a weighted average is taken of the samples taken at Earth at present Mars azimuth on the solar rotation before and after the sampling time at Mars. We refer to this corrected version of F10.7 as F10.7 Earth since it is taken at Earth orbit. Because the orbit of Earth is nearly circular, F10.7 Earth should be an index of the effect of solar activity on EUV flux without variation in the distance of Mars from the Sun. The square of the ratio of Mars to Earth heliocentric distances is applied to the azimuthally corrected result. We refer to this quantity as F10.7 Mars. Our correction of F10.7 for Mars orbit is similar to that used by Breus et al. [2004] for the related quantity E10.7. Note that our sampling interval occurs in the waning years of Solar Cycle 23, in which F10.7 Earth is always less than 105, while the upper limit of F10.7 Earth over a solar cycle is close to 300 . Thus all of our samples are at low values of intrinsic solar EUV flux. Much of the variation in our sampling of EUV flux is therefore due to the orbital variation in heliocentric distance $R$.

[56] To show the effect of varying EUV flux on the Martian ionosphere, the same procedure as in section 5.2 is used; the parameters resulting from individual good fits are ordered by F10.7 Mars, placed in bins of about 100, and averaged. The results for $n_{0}, h_{0}$, and $H$ as a function of F10.7 Mars are shown in Figures $8 \mathrm{a}-8 \mathrm{c}$. Figure $8 \mathrm{~d}$ shows the bin-averaged values of the solar zenith angle. The color coding and vertical and horizontal bars are as explained in section 5.2 .

[57] Figure 8a shows the result of this procedure for $n_{0}$ : an approximately monotonic increasing function. A regression analysis on $\ln n_{0}$ versus $\ln (\mathrm{F} 10.7)$ gives a value of $d \ln$ $n_{0} / d \ln$ F10.7 of $0.30 \pm 0.04$, the fit line here shown by a solid black line. Hantsch and Bauer [1990] report 0.36 for this exponent based on data from various Mariner spacecraft; Fox and Yeager [2006] derive values ranging from 0.29 to 0.41 at solar zenith angles from $60^{\circ}$ to $90^{\circ}$; Breus et al. [2004] get $0.37 \pm 0.06$. Our results are fairly close to those of previous work.

[58] Figure $8 \mathrm{~b}$ shows the results of the bin-averaging procedure for $h_{0}$. The values for Intervals 1 and 3 are some 15 to $20 \mathrm{~km}$ higher than for Interval 2; however, the transition is not smooth or monotonic.

[59] Figure 8c shows the results for the neutral scale height $H$. There is no apparent overall trend in $H$ with variation of F10.7 Mars. This result differs from those of Fox and Yeager [2006] and the models of Krasnopolsky [2002], which show an atmospheric temperature increase with increased solar activity.

[60] Figure 8d shows the variation of solar zenith angle for the F10.7 bins. Some of the detailed variation of the three Chapman parameters appears to correlate with changes in solar zenith angle. Low solar zenith angles appear to correlate with high $n_{0}$, high $h_{0}$, and low $H$. 

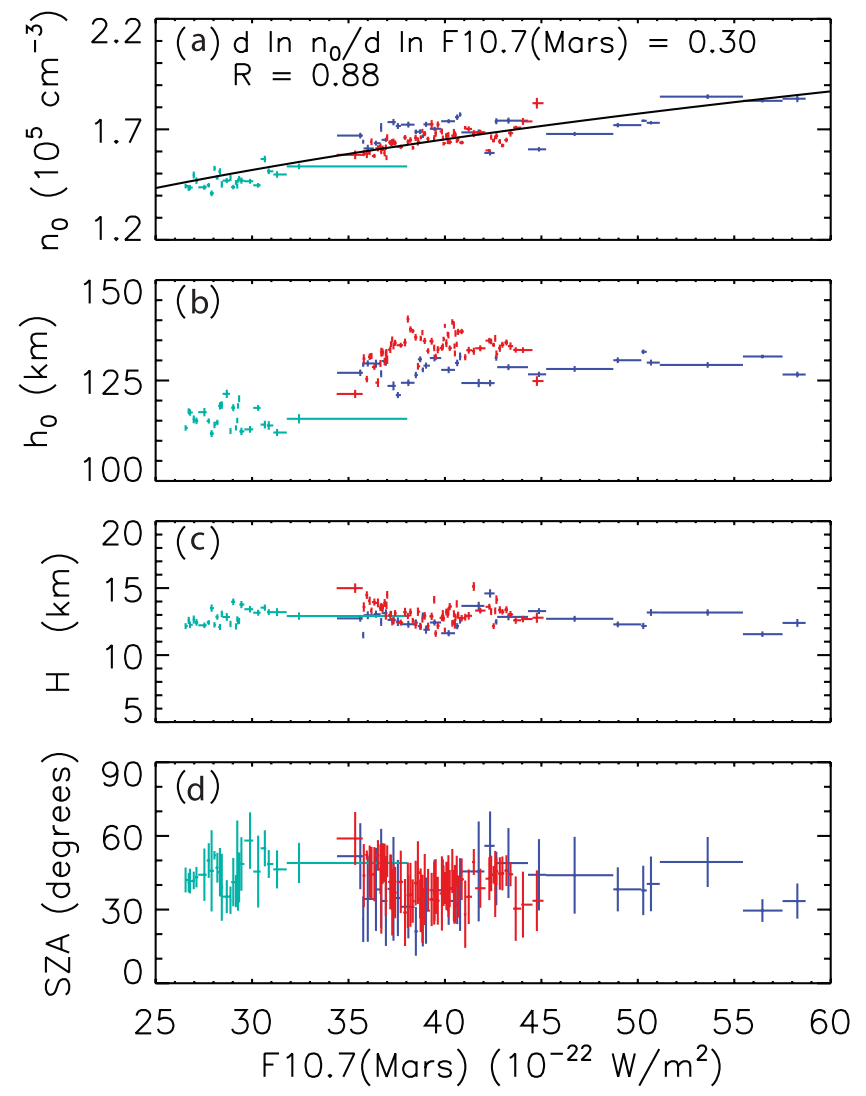

Figure 8. Bin averages of Chapman parameters and solar zenith angle as a function of F10.7 Mars. Colors stand for data intervals as given in section 5.2. (a) The variable $n_{0}$ displays a single increasing trend with $\mathrm{F} 10.7_{\text {Mars. }}$ A regression analysis gives $d \ln n_{0} / d \ln \mathrm{F} 10.7=0.30 \pm 0.04$, less than the result derived by Hantsch and Bauer [1990] but within the range of values given by Fox and Yeager [2006] for a range of solar zenith angles. (b) The variable $h_{0}$ does not vary monotonically over the range of F10.7. Intervals 1 and 3 have higher values than Interval 2 by $15-25 \mathrm{~km}$, consistent with higher values of F10.7 Mars during these intervals. (c) $H$ is approximately constant with F10.7 $7_{\text {Mars }}$. (d) Solar zenith angle does not show a large-scale bias; however, some of the smaller timescale variation of the Chapman parameters appears to correlate with variation of solar zenith angle.

\subsection{Variation With Solar Zenith Angle}

[61] Bin averages for the Chapman parameters and latitude as organized by solar zenith angle $\chi$, using the same method as in the previous two subsections, are shown in Figure 9, where $n_{0}$ is shown in Figure 9a, $h_{0}$ is shown in Figure $9 \mathrm{~b}, H$ is shown in Figure 9c, and latitude is shown in Figure 9d. Figure 9a indicates that $n_{0}$ is approximately constant for all values of $\chi$, consistent with the Chapman model, but with a possible drop off for $\chi>60^{\circ}$. Figure $9 \mathrm{~b}$ shows $h_{0}$ for all three intervals peaking slightly at about $\chi=$ $45^{\circ}$ and decreasing for $\chi>60^{\circ}$. Otherwise, the details of the variation of $h_{0}$ vary for the three intervals. Intervals 2 and 3 show slight increases toward the equator while Interval 1 appears to fall off. Interval 3 also has a peak near $\chi=60^{\circ}$ that does not occur in the other intervals. Unlike the approximately consistent behavior of $n_{0}, h_{0}$ varies in a way individual to each interval. According to Bougher et al. [2001] the profile peak altitude is sensitive to the temperature of the lower atmosphere, implying that $h_{0}$ may react strongly to local conditions such as Martian geography and dust storms [see also Wang and Nielsen, 2003b].

[62] Figure 9c shows the same behavior of $H$ for all three intervals: $H$ as a function of $\chi$ is constant at a value of $\sim 11 \mathrm{~km}$ for $\chi<40^{\circ}$. Intervals 1,2 , and 3 reach maxima of about 15 , 14 , and $17 \mathrm{~km}$ at $\chi \approx 70^{\circ}$. The near-peak temperature is related to the neutral scale height by the formula

$$
H=k_{\mathrm{B}} T /\left(m_{\mathrm{P}} A g_{\text {Mars }}\right)
$$

where $k_{\mathrm{B}}$ is Boltzmann's constant, $m_{\mathrm{P}}$ the mass of a proton, $A$ the average molecular mass of the Martian atmosphere (taken to be 44, approximately the molecular mass of $\mathrm{CO}_{2}$ ), and $g_{\text {Mars }}$ the acceleration due to gravity, approximately 3.4
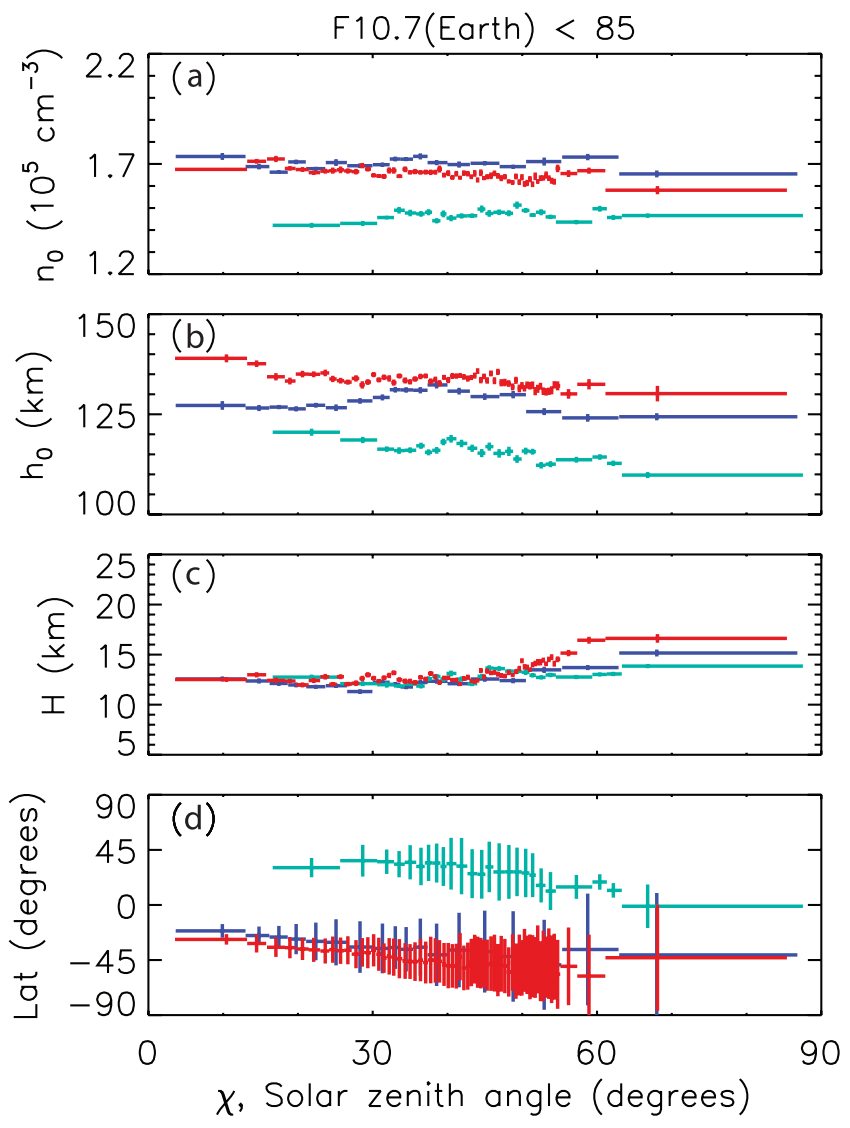

Figure 9. Bin averages of the Chapman parameters and latitude organized by solar zenith angle $\chi$. Colors stand for data intervals as given in section 5.2. (a) The variables $n_{0}$, (b) $h_{0}$, (c) $H$, and (d) latitude. In Figure 9a, $n_{0}$ for all three intervals is shown to vary only slightly out to $\chi=60^{\circ}$, after which a slight decrease in $n_{0}$ for all three intervals is evident. In Figure 9b, all three intervals are slightly peaked around $\chi=40^{\circ}-45^{\circ}$ and show a decrease for $\chi=60^{\circ}$. As $\chi$ approaches $0^{\circ}, h_{0}$ increases for Intervals 2 and 3 while decreasing for Interval 1, a discrepancy not explained by latitude sampling shown in Figure 9d. In Figure 9c, for all intervals, $H$ is constant at $\sim 11 \mathrm{~km}$ for $\chi<40^{\circ}$; $H$ then increases out to $\chi \approx 70^{\circ}$ to maxima of $14 \mathrm{~km}$ for Interval 1 , $15 \mathrm{~km}$ for Interval 2, and $17 \mathrm{~km}$ for Interval 3. 

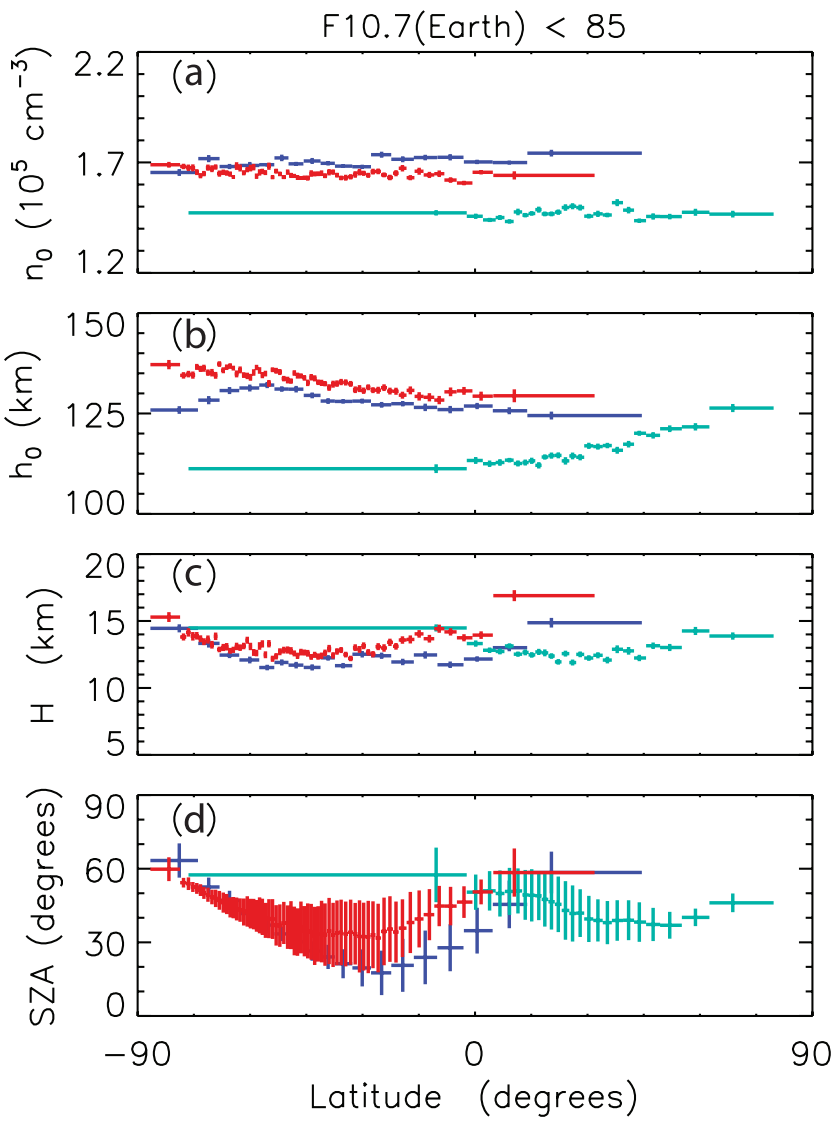

Figure 10. Bin averages of the Chapman parameters and $\chi$ organized by latitude. Colors stand for data intervals as given in section 5.2. (a) The variables $n_{0}$, (b) $h_{0}$, (c) $H$, (d) $\chi$. In Figure $10 \mathrm{a}, n_{0}$ shows little variation with latitude. In Figure $10 \mathrm{~b}, h_{0}$ increases from equatorial to high latitudes in both directions, with Interval 1 turning down below about $-70^{\circ}$. Figure 10c indicates that the behavior of $H$ is largely consistent with its being a function of $\chi$ as discussed in section 5.4. Figure 10d shows that the discrepancy between the Intervals 1 and 2 at high southern latitude is not the result of sampling differences in $\chi$.

$\mathrm{m} / \mathrm{s}^{2}$. The three near-terminator scale heights given above correspond to temperatures of $270 \mathrm{~K}$ (Interval 1, $1.39 \mathrm{AU}<$ $R<1.48 \mathrm{AU}$ ), $250 \mathrm{~K}$ (Interval 2, $1.57 \mathrm{AU}<R<1.67 \mathrm{AU}$ ), and $310 \mathrm{~K}$ (Interval 3, $1.38 \mathrm{AU}<R<1.42 \mathrm{AU}$ ). The nearterminator values of the near-peak temparature are seen to roughly correlate with the effect of heliocentric distance. Since our results are taken for F10.7 $7_{\text {Earth }}<85$, they should correspond with those reported in Table 3 of Fox and Yeager [2006] for low solar activity. These authors give a range of $H$ from 8.2 to $10.3 \mathrm{~km}$. Our results are some $70 \%$ higher but reveal the same upward trend with increasing values of F10.7 Earth.

[63] Figure 9d shows that differences between the three intervals are not due to sampling effects in latitude or, by implication, local time.

\subsection{Variation With Latitude}

[64] Bin averages for the Chapman parameters and solar zenith angle $\chi$ organized by latitude are shown in Figure 10, with Figure 10a showing results for $n_{0}$, Figure $10 \mathrm{~b}$ showing results for $h_{0}$, Figure 10c showing results for $H$, and Figure $10 \mathrm{~d}$ showing results for $\chi$. Figure 10a, similar to Figure 9a, indicates that $n_{0}$ undergoes only slight variation with latitude for each of the three intervals. In contrast Figure $9 \mathrm{~b}$, in Figure $10 \mathrm{~b}, h_{0}$ shows a steady increase toward high latitude in both directions, with Interval 1 turning down slightly at Lat $<-70$. The increase in $h_{0}$ towards high latitudes is in contrast to the decrease toward high solar zenith angles observed in Figure 9b. Bougher et al. [2001] have pointed out that the ionospheric peak altitude varies with the expansion and contraction of the lower atmosphere. The observed increase in peak altitude towards high latitudes during summer appears to be a seasonal effect, related to the thermal expansion and contraction of the lower atmosphere with varying insolation.

[65] In contrast to $h_{0}$, Figures 10c and 10d together show that the behavior of $H$ is consistent with the dependence of $H$ on $\chi$ as shown by Figure 9c.

\section{Near-Peak Temperatures Derived From the Neutral Scale Height}

[66] The Chapman model assumes an isothermal atmosphere, in which the temperature is related to the atmospheric scale height by equation (13). The atmosphere is actually not isothermal with change in either altitude or solar zenith angle; however, $H$ derived from the Chapman relation can be thought of as reflecting the atmospheric temperature locally, around the ionospheric electron density peak. In order to sum up the variation of our well-fit data over a variety of conditions, temperatures computed from our neutral scale heights $H$ by equation (13) have been binned by solar activity (as indexed by F10.7 Earth), season (indexed by $L_{\mathrm{S}}$ ), solar zenith angle, and northern or southern hemisphere. The bins in $L_{\mathrm{S}}$ are centered on perihelion and aphelion, and the median heliospheric distance for each $L_{\mathrm{S}}$ bin is given in the table notes. The results of this rebinning of our data is presented in Table 1.

[67] The table shows that near-peak temperature increases with solar zenith angle, consistent with Figure 9 and with the results of Fox and Yeager [2006]. The highest temperatures are seen at high solar zenith angles in the southern hemisphere near perihelion. During this time, temperatures in the northern hemisphere are also higher than at other times, particularly the northern hemisphere summer, near aphelion, a result that may reflect the warming trend observed in the northern hemisphere during winter detected by Keating et al. [2007] and modeled by Bougher et al. [2006]. Heliocentric distance appears to have more effect on near-peak temperature than does the season.

[68] Both Figure 9 and Table 1 indicate that variation in near-peak temperature is greatest at solar zenith angles greater than $60^{\circ}$. Table 1 indicates a variation of about $50 \mathrm{~K}$ in this solar zenith angle range in both hemispheres for F10.7 $7_{\text {Earth }}<85$. The higher bin in F10.7 $7_{\text {Earth }}$ does not have enough samples to make a comparison.

[69] In the southern hemisphere for $L_{\mathrm{S}}$ between $-68^{\circ}$ and $22^{\circ}$, varying the values of F10.7 $7_{\text {Earth }}$ from $65<\mathrm{F} 10.7_{\text {Earth }}<$ 85 to $85<$ F $10.7_{\text {Earth }}<105$ is associated with an increase of $15-25 \mathrm{~K}$ in near-peak temperature at solar zenith angles below $60^{\circ}$. At solar zenith angle greater than $60^{\circ}$ there is no apparent effect. For this comparison, the heliocentric dis- 
tance is around 1.5 AU and the variation in F10.7 Earth is much less than that between solar minimum and maximum. Thus, our variation of $50 \mathrm{~K}$ compares favorably with the modeling result of $110 \mathrm{~K}$ for solar minimum to maximum near aphelion and $150 \mathrm{~K}$ near perihelion.

[70] The near-peak temperatures shown by Table 1 vary from $220 \mathrm{~K}$ to about $300 \mathrm{~K}$, somewhat less than the exobase temperature variation of $200 \mathrm{~K}$ to $380 \mathrm{~K}$ reported by Bougher et al. [2000] at solar zenith angles between $45^{\circ}$ and $60^{\circ}$. The difference in variation can probably be accounted for by our sampling near the end of Solar Cycle 23 and therefore at values of F10.7 Earth less than 105. Thus, the results shown in this table appear to confirm the modeling results of Bougher et al. [2000] rather than the accelerometer measurements of Keating et al. [2007], which indicate little or no temperature variation in this region.

\section{Discussion and Interpretation}

[71] Ionospheric traces from 34,492 ionograms from the Mars Express ionospheric sounder have been accumulated. Of these, 14,060 traces have yielded acceptable inversions and 12,291 have yielded acceptable fits to the Chapman ionospheric model. These results have been analyzed to correlate the three free parameters of the Chapman model with the following variable inputs: the presence or absence of solar energetic particles, EUV flux represented by F10.7 Mars, Martian season represented by $L_{S}$, solar zenith angle $\chi$, and Mars latitude. These analyses yield inferences regarding behavior of the Martian atmosphere and ionosphere.

[72] In interpreting these results it is useful to recall some simple physical correspondences. First, $n_{0}$ varies with the square root of the ion production rate [Bougher et al., 2001], which will increase with EUV intensity or with an increase in ionizing particle impacts. Second, $h_{0}$ varies with the thermal expansion or contraction of the lower atmosphere [Bougher et al., 2001; Bougher et al., 2006]. The sensitivity of $h_{0}$ to the temperature of the lower atmosphere causes it reflect local phenomena more than the other parameters. Finally, the neutral scale height $H$ is related to the neutral atmospheric temperature at the altitude of the ionospheric peak by equation (13).

[73] Our analyses yield the following results:

[74] 1. The occurrence of solar energetic particles is associated with an increase in $n_{0}$ of $6 \%$, an increase in $h_{0}$ of $3 \mathrm{~km}$, and, a decrease in $H$ of $0-7 \mathrm{~km}$. The increase $n_{0}$ is expected because of impact ionization. A full understanding of the possible changes in peak altitude and scale height will require a modeling of the interaction of the energetic particles with the Martian atmosphere.

[75] 2. F10.7 Mars organizes $n_{0}$ as a smoothly increasing function, varying from $1.4 \times 10^{5}$ to $1.8 \times 10^{5} \mathrm{~cm}^{-3}$ as F10.7 Earth varies between 65 and 105 and $R$ varies between 1.38 AU and 1.67 AU. A value of $d \ln n_{0} / d \ln$ F10.7 Mars $=$ $0.30 \pm 0.04$, is derived, lower than those of Hantsch and Bauer [1990] and Breus et al. [2004] but bracketed by values derived by Fox and Yeager [2006]. Discrepancies may reflect sampling limited to periods of low solar activity toward the end of Solar Cycle 23. Breus et al. [2004] note higher values of $n_{0}$ for lower values of Mars-corrected
E10.7 (see their Table 1) when their data are broken down by northern or southern hemisphere. They attribute this discrepancy with the general upward trend of $n_{0}$ with radiative input to the influence of hot electrons inhabiting the minimagnetospheres prevalent in the southern hemisphere of Mars. They are able to make this comparison because of the existence of a sizable body of data in which the northern hemisphere receives stronger insolation than the southern. We are unable to make a comparison with this result because virtually all of our northern hemisphere data (the blue-green colored points in Figure 8a) are at lower values of F10.7 Mars than our southern hemisphere data (red and blue points).

[76] 3. The peak altitude $h_{0}$ varies between Interval 2 (1.57AU $<R<1.67 \mathrm{AU})$ at about $115 \mathrm{~km}$ and combined Intervals 1 (1.39 AU $<R<1.48 \mathrm{AU})$ and $3(1.38 \mathrm{AU}<R<$ $1.42 \mathrm{AU})$, at about $135 \mathrm{~km}$. The variation is not smooth, probably because of the dependence of $h_{0}$ on local conditions. It can be seen from Figure $7 \mathrm{f}$ that Interval 3, which has the highest values of $h_{0}$, is sampled at high latitudes in the period surrounding perihelion. The neutral scale height $H$ remains approximately constant with F10.7 Mars over the three intervals, in contrast with results of, e.g., Fox and Yeager [2006].

[77] 4. The inferred subsolar peak density $n_{0}$ does not appear to be a strong function of the solar zenith angle out to about $60^{\circ}$, implying that the Chapman model effectively describes the variation of peak density out to this value.

[78] 5. At low latitudes the electron density peak altitude $h_{0}$ decreases with high values of the solar zenith angle due to oblique insolation and consequent weak heating of the lower atmosphere.

[79] 6. In contrast to the previous point, at high latitudes (implying high solar zenith angle) near summer solstice, $h_{0}$ is elevated. The ionospheric peak is known to respond strongly to the temperature of the lower atmosphere [Bougher et al., 2001]. It is likely that increased insolation during summer at high latitudes is effective in warming the lower atmosphere, possibly concentrated by increased atmospheric dust.

[80] 7. The neutral scale height $H$ as a function of solar zenith angle is constant at an approximate value of $11 \mathrm{~km}$ from 0 to $\sim 40^{\circ}$, with nearly the same values for the three sampling intervals. Above solar zenith angles of $40^{\circ}$ the average value of $H$ increases smoothly to a high-solar zenith angle maximum of $14 \mathrm{~km}$ for times around aphelion, $17 \mathrm{~km}$ for times around perihelion, and $15 \mathrm{~km}$ for times in between, corresponding to near-peak temperatures of $270 \mathrm{~K}, 250 \mathrm{~K}$, and $310 \mathrm{~K}$. These temperatures correlate roughly with the range of heliocentric distance of the three data intervals as given above. The variation in temperature for $\chi>40^{\circ}$ is less than that found by Bougher et al. [2000] but probably still consistent with their modeling results, because of our sampling during a period of low solar activity. The increase in $H$ with increasing $\chi$ is consistent with the dependence seen by Fox and Yeager [2006], cited in their Table 5, although our values are somewhat greater than theirs.

[81] The lack of expected variation of $H$ with EUV flux is somewhat troubling and may point to one of several problems with our method. First, a more precise ionospheric model than the Chapman model may be called for. Second, 
the lack of data at low sounding frequencies coupled with the assumption of an exponential functional form may have an effect on our ability to determine scale height. A better choice of function might improve our sensitivity. Nonetheless, the method in use is sensitive enough to detect predicted variation of $H$ with solar zenith angle, so it is unclear why other variability is not detected.

[82] We have here presented results of analysis using ionospheric traces generated by the MARSIS ionospheric sounder, inverted to make electron density profiles, and fit to a Chapman layer, for 2 years of data. The statistical results of these analyses are broadly coherent with previous results; however, there are many details and some discrepancies yet to be explained. We hope in future to improve our inversion technique and also to apply a more precise ionospheric model to these data. Also we hope to fill in gaps in our data set, acquiring more data around the winter solstices in both hemispheres and in the dawn sector. Other goals are to gauge the effects of crustal magnetic fields, varying solar wind pressure, and the occurrence of dust storms on the characteristics of the principal ionization layer. All should contribute to a comprehensive understanding of the Martian system.

[83] Acknowledgments. We would like to thank Zeynep Sagtas Bilki, Douglas Karkow, Andrew Kopf, Don Kirchner, and Julia Nelson for their meticulous work in processing a very large number of ionogram traces. We would also like to thank Paul Withers for help in accessing MGS Radio Science electron density profiles and Martin Pätzold for providing Mars Express Radio Science electron density profiles. This work was supported under JPL contract 1224107.

[84] Wolfgang Baumjohann thanks the reviewers for their assistance in evaluating this paper.

\section{References}

Bougher, S. W., S. Engel, R. G. Roble, and B. Foster (2000), Comparative terrestrial planet thermospheres 3. solar cycle variation of global structure and winds at solstices, J. Geophys. Res., 105, 17,669-17,692.

Bougher, S. W., S. Engel, D. P. Hinson, and J. M. Forbes (2001), Mars Global Surveyor Radio Science electron density profiles: Neutral atmosphere implications, Geophys. Res. Lett., 28, 3091-3094.

Bougher, S. W., J. M. Bell, J. R. Murphy, M. A. Lopez-Valverde, and P. G. Withers (2006), Polar warming in the Mars thermosphere: seasonal variations owing to changing insolation and dust distributions, Geophys. Res. Lett., 33, L02203, doi:10.1029/2005GL024059.

Breus, T. K., A. M. Krymskii, D. H. Crider, N. F. Ness, D. P. Hinson, and K. K. Barashyan (2004), Effect of the solar radiation in the topside atmosphere/ionosphere of Mars: Mars Global Surveyor observations, J. Geophys. Res., 109, A09310, doi:10.1029/2004JA010431.

Budden, K. G. (1961), Radio Waves in the Ionosphere, Cambridge Univ. Press, Cambridge, U. K.

Chapman, S. (1931a), The absorption and dissociative or ionizing effect of monochromatic radiation in an atmosphere on a rotating Earth, Proc. Phys. Soc., 43, 26-45.

Chapman, S. (1931b), The absorption and dissociative or ionizing effect of monochromatic radiation in an atmosphere on a rotating Earth, part ii. grazing incidence, Proc. Phys. Soc., 43, 483-501.

Chicarro, A., P. Martin, and R. Trautner (2004), The Mars Express mission: An overview, in Mars Express: A European Mission to the Red Planet, edited by A. Wilson, pp. 3-16, ESA Publ. Div., Noordwijk, Netherlands.

Duru, F., D. A. Gurnett, T. F. Averkamp, D. L. Kirchner, R. L. Huff, A. M. Persoon, J. J. Plaut, and G. Picardi (2006), Magnetically controlled structures in the ionosphere of Mars, J. Geophys. Res., 111, A12204, doi:10.1029/2006JA011975.

Duru, F., D. A. Gurnett, D. D. Morgan, R. Modolo, A. F. Nagy, and D. Najib (2008), Electron densities in the upper ionosphere of Mars from the excitation of electron plasma oscillations, J. Geophys. Res., 113, A07302, doi:10.1029/2008JA013073.

Espley, J. R., W. M. Farrell, D. A. Brain, D. D. Morgan, B. Cantor, J. J. Plaut, M. H. Acuña, and G. Picardi (2007), Absorption of MARSIS radar signals: Solar energetic particles and the daytime ionosphere, Geophys. Res. Lett., 34, L09101, doi:10.1029/2006GL028829.

Fox, J. L., and K. E. Yeager (2006), Morphology of the near-terminator Martian ionosphere: a comparison of models and data, J. Geophys. Res., 111, A10309, doi:10.1029/2006JA011697.

Gurnett, D. A., and A. Bhattacharjee (2005), Introduction to Plasma Physics, Cambridge Univ. Press, Cambridge, U. K.

Gurnett, D. A., D. L. Kirchner, R. L. Huff, D. D. Morgan, A. M. Persoon, T. F. Averkamp, F. Duru, E. Nielsen, S. J. J. A.Plaut, and G. Picardi (2005), Radar soundings of the ionosphere of Mars, Science, 310, 1929-1933.

Gurnett, D. A., et al. (2008), An overview of radar soundings of the Martian ionosphere from the Mars Express spacecraft, Adv. Space Res., 41(9), 1335-1346, doi:10.1016/j.asr.2007.01.062.

Hantsch, M. H., and S. J. Bauer (1990), Solar control of the Mars ionosphere, Planet. Space Sci., 38, 539-542.

Hinson, D. P., R. A. Simpson, J. D. Twicken, G. L. Tyler, and F. M. Fasar (1999), Initial results from radio occultation measurements with Mars Global Surveyor, J. Geophys. Res., 104, 26,997-27,012.

Jackson, J. E. (1969), The reduction of topside ionograms to electrondensity profiles, Proc. IEEE, 57, 960-975.

Keating, G. M., S. W. Bougher, M. E. Theriot, and R. H. Tolson (2007), The Mars global thermosphere measured by accelerometer facilities aboard orbiters and properties of the exosphere, paper presented at European Mars Science and Exploration Conference: Mars Express and ExoMars, ESTEC, Noordwijk, Netherlands.

Kliore, A. J. (1992), Radio occultation observations of the ionospheres of Mars and Venus, in Venus and Mars: Atmospheres, Ionospheres, and Solar Wind Interactions, Geophys. Monogr. Ser, vol. 66, edited by J. G. Luhmann, M. Tatrallyay, and R. O. Pepin, pp. 265-276, AGU, Washington, D. C.

Krasnopolsky, V. A. (2002), Mars' upper atmosphere and ionosphere at low, medium and high solar activities: implications for evolution of water, J. Geophys. Res., 107(E12), 5128, doi:10.1029/2001JE001809.

Krymskii, A. M., N. F. Ness, D. H. Crider, T. K. Breus, M. H. Acuña, and D. P. Hinson (2004), Solar wind interaction with the ionosphere/atmosphere and crustal magnetic fields at Mars: Mars Global Surveyor Magnetometer/Electron Reflectometer, Radio Science, and Accelerometer data, J. Geophys. Res., 109, A11306, doi:10.1029/2004JA010420.

Manning, L. A. (1947), The determination of ionospheric electron distribution, Proc. IEEE, 35, 1203-1207.

Martinis, C. R., J. K. Wilson, and M. J. Mendillo (2003), Modeling day-today ionospheric variability on Mars, J. Geophys. Res., 108(A10), 1383, doi:10.1029/2003JA009973.

Morgan, D. D., D. A. Gurnett, D. L. Kirchner, R. L. Huff, D. A. Brain, W. V. Boynton, M. H. Acuña, J. J. Plaut, and G. Picardi (2006), Solar control of radar wave absorption by the Martian ionosphere, Geophys. Res. Lett., 33, L13202, doi:10.1029/2006GL026637.

Nielsen, E. (2004), Mars Express and MARSIS, Space Sci. Rev., 111, 245 262.

Nielsen, E., H. Zou, D. A. Gurnett, D. L. Kirchner, D. D. Morgan, R. Huff, R. Orosei, A. Safaeinili, J. J. Plaut, and G. Picardi (2006), Observations of vertical reflections from the topside Martian ionosphere, Space Sci. Rev., 126, 373-388, doi:10.1007/s11214-006-9113-y.

Nielsen, E., D. D. Morgan, D. L. Kirchner, J. J. Plaut, and G. Picardi (2007a), Absorption and reflection of radio waves in the Martian ionosphere, Planet. Space Sci., 55, 864-870, doi:10.1016/j.pss.2006.10.005. Nielsen, E., X.-D. Wang, D. A. Gurnett, D. L. Kirchner, R. Huff, R. Orosei, A. Safaeinili, J. J. Plaut, and G. Picardi (2007b), Vertical sheets of dense plasma in the topside Martian ionosphere, J. Geophys. Res., 112, E02003, doi:10.1029/2006JE002723.

Pätzold, M., S. Tellman, B. Haeusler, D. Hinson, R. Schaa, and G. L. Tyler (2005), A sporadic third layer in the ionosphere of Mars, Science, 310, $837-839$.

Pesnell, W. D., and G. Grebowsky (2000), Meteoric magnesium ions in the Martian atmosphere, J. Geophys. Res., 105, 1695-1707.

Picardi, G. et al. (2004), MARSIS: Mars Advanced Radar for Subsurface and Ionosphere Sounding, in Mars Express: A European Mission to the Red Planet, edited by A. Wilson, pp. 51-69, ESA Publ. Div., Noordwijk, Netherlands.

Picardi, G., et al. (2005), Radar soundings of the subsurface of Mars, Science, 310, 1925-1928.

Plaut, J. J., et al. (2007), Subsurface radar sounding of the south polar layered deposits of Mars, Science, 316(92), 5821, doi:10.1126/ science. 1139672.

Rishbeth, H., and O. K. Garriott (1969), Introduction to Ionospheric Physics, Academic, New York.

Rishbeth, H., and M. Mendillo (2004), Ionospheric layers of Earth and Mars, Planet. Space Sci., 52(9), 849-852.

Safaeinili, A., W. Kofman, J. Mouginot, Y. Gim, A. Herique, A. B. Ivanov, J. J. Plaut, and G. Picardi (2007), Estimation of the total electron content 
of the Martian ionosphere using radar sounder surface echoes, Geophys. Res. Lett., 34, L23204, doi:10.1029/2007GL032154.

Schröter, J., B. Heger, F. Steinhilber, and M. B. Kallenrode (2006), Energetic particles in the atmosphere: A monte-carlo simulation, Adv. Space Res., 37, 1597-1601, doi:10.1016/j.asr.2005.05.085.

Schunk, R. W., and A. F. Nagy (2000), Ionospheres: Physics, Plasma Physics, and Chemistry, Cambridge Univ. Press, Cambridge, U. K.

Smith, F. L., and C. Smith (1972), Numerical evaluation of Chapman's grazing incidence integral $\operatorname{ch}(x, \chi), J$. Geophys. Res., 77, 3592-3597.

Wang, J.-S., and E. Nielsen (2003a), Wavelike structures in the Martian topside ionosphere observed by Mars Global Surveyor, J. Geophys. Res., 108(E7), 5078, doi:10.1029/2003JE002078.

Wang, J.-S., and E. Nielsen (2003b), Behavior of the Martian dayside electron density peak during global dust storms, Planet. Space Sci., 51, 329-338, doi:10.1016/S0032-0633(03)00015-1.

Whittaker, E. T., and G. N. Watson (1927), A Course of Modern Analysis, Cambridge Univ. Press, Cambridge, U. K.

Wilson, A., (Ed.) (2004), Mars Express: A European Mission to the Red Planet, ESA Publ. Div., Noordwijk, Netherlands.
Withers, P., and M. Mendillo (2005), Response of peak electron densities in the Martian ionosphere to day-to-day changes in solar flux due to solar rotation, Planet. Space Sci., 53, 1401-1418, doi:10.1016/ j.pss.2005.07.010.

Zou, H., J.-S. Wang, and E. Nielsen (2005), Effect of the seasonal variations in the lower atmosphere on the altitude of the ionospheric main peak at Mars, J. Geophys. Res., 110, A09311, doi:10.1029/2004JA010963.

Zou, H., J.-S. Wang, and E. Nielsen (2006), Reevaluating the relationship between the Martian ionospheric peak density and the solar radiation, J. Geophys. Res., 111, A07305, doi:10.1029/2005JA011580.

J. L. Fox, Department of Physics, Wright State University, 3640 Colonel Glenn Highway, Dayton, OH 45435, USA

D. A. Gurnett, D. L. Kirchner, and D. D. Morgan, Department of Physics and Astronomy, University of Iowa, 718 Van Allen Hall, Iowa City, IA 52242, USA. (david-morgan@uiowa.edu)

E. Nielsen, Max Plank Institute for Solar System Studies, D-37189 Katlenburg-Lindau, Germany.

J. J. Plaut, Jet Propulsion Laboratory, 4800 Oak Grove Drive, Pasadena, CA 91109, USA. 